\title{
The Wiener-Hopf Equation Technique for Solving General Nonlinear Regularized Nonconvex Variational Inequalities
}

\author{
Javad Balooee ${ }^{1}$ Yeol Je $\mathrm{Cho}^{2^{*}}$ and Mee Kwang Kang ${ }^{3^{*}}$
}

\author{
* Correspondence: yjcho@gnu.ac.kr; \\ mee@deu.ac.kr \\ ${ }^{2}$ Department of Mathematics \\ Education and the RINS \\ Gyeongsang National University, \\ Chinju 660-701, Korea \\ ${ }^{3}$ Department of Mathematics, \\ Dongeui University Pusan 614-714, \\ Korea \\ Full list of author information is \\ available at the end of the article
}

\begin{abstract}
In this paper, we introduce and study some new classes of extended general nonlinear regularized non-convex variational inequalities and the extended general nonconvex Wiener-Hopf equations, and by the projection operator technique, we establish the equivalence between the extended general nonlinear regularized nonconvex variational inequalities and the fixed point problems as well as the extended general nonconvex Wiener-Hopf equations. Then by using this equivalent formulation, we discuss the existence and uniqueness of solution of the problem of extended general nonlinear regularized nonconvex variational inequalities. We apply the equivalent alternative formulation and a nearly uniformly Lipschitzian mapping $S$ for constructing some new $p$-step projection iterative algorithms with mixed errors for finding an element of set of the fixed points of nearly uniformly Lipschitzian mapping $S$ which is unique solution of the problem of extended general nonlinear regularized nonconvex variational inequalities. We also consider the convergence analysis of the suggested iterative schemes under some suitable conditions.

Mathematics Subject Classification (2010)

Primary 47H05; Secondary 47J20, $49 J 40$

Keywords: variational inequalities, fixed point problems, prox-regularity, nearly uniformly Lipschitzian mappings, p-step projection iterative algorithms, extended general nonconvex Wiener-Hopf equations, convergence analysis
\end{abstract}

\section{Introduction}

The theory of variational inequalities, which was initially introduced by Stampacchia [1] in 1964, is a branch of the mathematical sciences dealing with general equilibrium problems. It has a wide range of applications in economics, optimizations research, industry, physics, and engineering sciences. Many research papers have been written lately, both on the theory and applications of this field. Important connections with main areas of pure and applied sciences have been made, see for example [2,3] and the references cited therein. The development of variational inequality theory can be viewed as the simultaneous pursuit of two different lines of research. On the one hand, it reveals the fundamental facts on the qualitative aspects of the solution to important classes of problems; on the other hand, it also enables us to develop highly efficient and powerful new numerical methods to solve, for example, obstacle, unilateral, free, moving and the complex equilibrium problems. One of the most interesting and

\section{Springer}

(C) 2011 Balooee et al; licensee Springer. This is an Open Access article distributed under the terms of the Creative Commons Attribution License (http://creativecommons.org/licenses/by/2.0), which permits unrestricted use, distribution, and reproduction in any medium, provided the original work is properly cited. 
important problems in variational inequality theory is the development of an efficient numerical method. There is a substantial number of numerical methods including projection method and its variant forms, Wiener-Holf (normal) equations, auxiliary principle, and descent framework for solving variational inequalities and complementarity problems. For the applications on physical formulations, numerical methods and other aspects of variational inequalities, see [1-52] and the references therein.

Projection method and its variant forms represent important tool for finding the approximate solution of various types of variational and quasi-variational inequalities, the origin of which can be traced back to Lions and Stampacchia [31]. The projection type methods were developed in 1970's and 1980's. The main idea in this technique is to establish the equivalence between the variational inequalities and the fixed point problems using the concept of projection. This alternate formulation enables us to suggest some iterative methods for computing the approximate solution. Shi [50,51] and Robinson [48] considered the problem of solving a system of equations which are called the Wiener-Hopf equations or normal maps. Shi [50] and Robinson [48] proved that the variational inequalities and the Wiener-Hopf equations are equivalent by using the projection technique. It turned out that this alternative equivalent formulation is more general and flexible. It has shown in [48-53] that the Wiener-Hopf equations provide us a simple, elegant and convenient device for developing some efficient numerical methods for solving variational inequalities and complementarity problems.

It should be pointed that almost all the results regarding the existence and iterative schemes for solving variational inequalities and related optimizations problems are being considered in the convexity setting. Consequently, all the techniques are based on the properties of the projection operator over convex sets, which may not hold in general, when the sets are nonconvex. It is known that the uniformly prox-regular sets are nonconvex and include the convex sets as special cases, for more details, see for example $[23,28,29,46]$. In recent years, Bounkhel et al. [23], Noor [36,41] and Pang et al. [45] have considered variational inequalities in the context of uniformly prox-regular sets.

On the other hand, related to the variational inequalities, we have the problem of finding the fixed points of the nonexpansive mappings, which is the subject of current interest in functional analysis. It is natural to consider a unified approach to these two different problems. Motivated and inspired by the research going in this direction, Noor and Huang [43] considered the problem of finding the common element of the set of the solutions of variational inequalities and the set of the fixed points of the nonexpansive mappings. Noor [38] suggested and analyzed some three-step iterative algorithms for finding the common elements of the set of the solutions of the Noor variational inequalities and the set of the fixed points of nonexpansive mappings. He also discussed the convergence analysis of the suggested iterative algorithms under some conditions.

Recently, Qin and Noor [47] established the equivalence between general variational inequalities and general Wiener-Hopf equations. They proposed and analyzed a new iterative method for solving variational inequalities and related optimization problems. They also considered the problem of finding a comment element of fixed points of nonexpansive mappings and the set of solution of the general variational inequalities. 
It is well known that every nonexpansive mapping is a Lipschitzian mapping. Lipschitzian mappings have been generalized by various authors. Sahu [53] introduced and investigated nearly uniformly Lipschitzian mappings as generalization of Lipschitzian mappings.

Motivated and inspired by the above works, at the present paper, some new classes of the extended general nonlinear regularized nonconvex variational inequalities and the extended general nonconvex Wiener-Hopf equations are introduced and studied, and by the projection technique, the equivalence between the extended general nonlinear regularized nonconvex variational inequalities and the fixed point problems as well as the extended general nonconvex Wiener-Hopf equations is proved. Then by using this equivalent formulation, the existence and uniqueness of solution of the problem of extended general nonlinear regularized nonconvex variational inequalities are discussed. Applying the equivalent alternative formulation and a nearly uniformly Lipschitzian mapping $S$, some new $p$-step projection iterative algorithms with mixed errors for finding an element of the set of fixed points of nearly uniformly Lipschitzian mapping $S$ which is a unique solution of the problem of extended general nonlinear regularized nonconvex variational inequalities are defined. The convergence analysis of the suggested iterative schemes under some suitable conditions is discussed. Some remarks about established statements by Noor [38], Noor et al. [44] and Qin and Noor [47] are presented. Also, this fact that their statements are special cases of our results is shown. The results obtained in this paper may be viewed as an refinement and improvement of the previously known results.

\section{Preliminaries and basic results}

Throughout this article, we will let $\mathcal{H}$ be a real Hilbert space which is equipped with an inner product $\langle.,$.$\rangle and corresponding norm \|c \operatorname{dot}\|$ and $K$ be a nonempty convex subset of $\mathcal{H}$. We denote by $d_{K}(\cdot)$ or $d(., K)$ the usual distance function to the subset $K$, i.e., $d_{K}(u)=\inf _{v \in K}\|u-v\|$. Let us recall the following well-known definitions and some auxiliary results of nonlinear convex analysis and nonsmooth analysis [27-29,46].

Definition 2.1. Let $u \in \mathcal{H}$ is a point not lying in $K$. A point $v \in K$ is called $a$ closest point or a projection of $u$ onto $K$ if $d_{K}(u)=\|u-v\|$. The set of all such closest points is denoted by $P_{K}(u)$, i.e.,

$$
P_{K}(u):=\left\{v \in K: d_{K}(u)=\|u-v\|\right\} .
$$

Definition 2.2. The proximal normal cone of $K$ at a point $u \in \mathcal{H}$ with $u \notin K$ is given by

$$
N_{K}^{P}(u):=\left\{\xi \in \mathcal{H}: u \in P_{K}(u+\alpha \xi) \text { for some } \alpha>0\right\} .
$$

Clarke et al. [28], in Proposition 1.1.5, give a characterization of $N_{K}^{P}(u)$ as follows:

Lemma 2.3. Let $K$ be a nonempty closed subset in $\mathcal{H}$. Then $\xi \in N_{K}^{P}(u)$ if and only if there exists a constant $\alpha=\alpha(\xi, u)>0$ such that $\langle\xi, v-u\rangle \leq \alpha\|v-u\|^{2}$ for all $v \in K$.

The above inequality is called the proximal normal inequality. The special case in which $K$ is closed and convex is an important one. In Proposition 1.1.10 of [28], the authors give the following characterization of the proximal normal cone the closed and convex subset $K \subset \mathcal{H}$ : 
Lemma 2.4. Let $K$ be a nonempty closed and convex subset in $\mathcal{H}$. Then $\xi \in N_{K}^{P}(u)$ if and only if $\langle\xi, v-u\rangle \leq 0$ for all $v \in K$.

Definition 2.5. Let $X$ is a real Banach space and $f: X \rightarrow \mathbb{R}$ be Lipschitzian with constant $\tau$ near a given point $x \in X$, that is, for some $\varepsilon>0$, we have $|f(y)-f(z)| \leq \tau|| y-z||$ for all $y, z \in B(x ; \varepsilon)$, where $B(x ; \varepsilon)$ denotes the open ball of radius $r>0$ and centered at $x$. The generalized directional derivative of $f$ at $x$ in the direction $v$, denoted by $f(x ; v)$, is defined as follows:

$$
f^{\circ}(x ; v)=\limsup _{y \rightarrow x, t \downarrow 0} \frac{f(y+t v)-f(\gamma)}{t},
$$

where $y$ is a vector in $X$ and $t$ is a positive scalar.

The generalized directional derivative defined earlier can be used to develop a notion of tangency that does not require $K$ to be smooth or convex.

Definition 2.6. The tangent cone $T_{K}(x)$ to $K$ at a point $x$ in $K$ is defined as follows:

$$
T_{K}(x):=\left\{v \in \mathcal{H}: d_{K}^{\circ}(x ; v)=0\right\} .
$$

Having defined a tangent cone, the likely candidate for the normal cone is the one obtained from $T_{K}(x)$ by polarity. Accordingly, we define the normal cone of $K$ at $x$ by polarity with $T_{K}(x)$ as follows:

$$
N_{K}(x):=\left\{\xi:\langle\xi, v\rangle \leq 0, \quad \forall v \in T_{K}(x)\right\} .
$$

Definition 2.7. The Clarke normal cone, denoted by $N_{K}^{C}(x)$, is given by $N_{K}^{C}(x)=\overline{c o}\left[N_{K}^{P}(x)\right]$, where $\overline{c o}[S]$ means the closure of the convex hull of $S$. It is clear that one always has $N_{K}^{P}(x) \subseteq N_{K}^{C}(x)$. The converse is not true in general. Note that $N_{K}^{C}(x)$ is always closed and convex cone, whereas $N_{K}^{P}(x)$ is always convex, but may not be closed (see $[27,28,46])$.

In 1995, Clarke et al. [29] introduced and studied a new class of nonconvex sets called proximally smooth sets; subsequently, Poliquin et al. in [46] investigated the aforementioned sets, under the name of uniformly prox-regular sets. These have been successfully used in many nonconvex applications in areas such as optimizations, economic models, dynamical systems, differential inclusions, etc. For such as applications see $[20-22,24]$. This class seems particularly well suited to overcome the difficulties which arise due to the nonconvexity assumptions on $K$. We take the following characterization proved in [29] as a definition of this class. We point out that the original definition was given in terms of the differentiability of the distance function (see [29]).

Definition 2.8. For any $r \in(0,+\infty]$, a subset $K_{r}$ of $\mathcal{H}$ is called normalized uniformly prox-regular (or uniformly $r$-prox-regular [29]) if every nonzero proximal normal to $K_{r}$ can be realized by an $r$-ball.

This means that for all $\bar{x} \in K_{r}$ and $0 \neq \xi \in N_{K_{r}}^{P}(\bar{x})$ with $\|\xi\|=1$,

$$
\langle\xi, x-\bar{x}\rangle \leq \frac{1}{2 r}\|x-\bar{x}\|^{2}, \quad \forall x \in K_{r} .
$$

Obviously, the class of normalized uniformly prox-regular sets is sufficiently large to include the class of convex sets, $p$-convex sets, $C^{1,1}$ submanifolds (possibly with 
boundary) of $\mathcal{H}$, the images under a $C^{1,1}$ diffeomorphism of convex sets and many other nonconvex sets, see [25,29].

Lemma 2.9. [29]A closed set $K \subseteq \mathcal{H}$ is convex if and only if it is proximally smooth of radius $r$ for every $r>0$.

If $r=+\infty$, then in view of Definition 2.8 and Lemma 2.9, the uniform $r$-prox-regularity of $K_{r}$ is equivalent to the convexity of $K_{r}$, which makes this class of great importance. For the case of that $r=+\infty$, we set $K_{r}=K$.

The following proposition summarizes some important consequences of the uniform prox-regularity needed in the sequel. The proof of this results can be found in $[29,46]$.

Proposition 2.10. Let $r>0$ and $K_{r}$ be a nonempty closed and uniformly r-prox-regular subset of $\mathcal{H}$. Set $U(r)=\left\{u \in \mathcal{H}: 0<d_{K_{r}}(u)<r\right\}$. Then the following statements hold:

(a) For all $x \in U(r)$, one has $P_{K_{r}}(x) \neq \emptyset$;

(b) For all $r^{\prime} \in(0, r), P_{K_{r}}$ is Lipschitzian continuous with constant $\frac{r}{r-r^{\prime}}$ on $U\left(r^{\prime}\right)=\left\{u \in \mathcal{H}: 0<d_{K_{r}}(u)<r^{\prime}\right\}$;

(c) The proximal normal cone is closed as a set-valued mapping.

As a direct consequent of part (c) of Proposition 2.10, we have $N_{K_{r}}^{C}(x)=N_{K_{r}}^{P}(x)$. Therefore, we will define $N_{K_{r}}(x):=N_{K_{r}}^{C}(x)=N_{K_{r}}^{P}(x)$ for such a class of sets.

In order to make clear the concept of $r$-prox-regular sets, we state the following concrete example: The union of two disjoint intervals $[a, b]$ and $[c, d]$ is $r$-prox-regular with $r=\frac{c-b}{2}$. The finite union of disjoint intervals is also $r$-prox-regular and $r$ depends on the distances between the intervals.

Definition 2.11. Let $T, g: \mathcal{H} \rightarrow \mathcal{H}$ be two single-valued operators. Then the operator $T$ is said to be:

(a) monotone if

$$
\langle T(x)-T(y), x-y\rangle \geq 0, \quad \forall x, y \in \mathcal{H} ;
$$

(b) $r$-strongly monotone if there exists a constant $r>0$ such that

$$
\langle T(x)-T(y), x-y\rangle \geq r\|x-y\|^{2}, \quad \forall x, y \in \mathcal{H} ;
$$

(c) $\kappa$-strongly monotone with respect to $g$ if there exists a constant $\kappa>0$ such that

$$
\langle T(x)-T(y), g(x)-g(y)\rangle \geq \kappa|| x-y \|^{2}, \quad \forall x, y \in \mathcal{H} ;
$$


(d) $(\xi, \varsigma)$-relaxed co-coercive if there exist constants $\xi, \varsigma>0$ such that

$$
\langle T(x)-T(y), x-y\rangle \geq-\xi\|T(x)-T(y)\|^{2}+\varsigma\|x-y\|^{2}, \quad \forall x, y \in \mathcal{H} ;
$$

(e) $\gamma$-Lipschitzian continuous if there exists a constant $\gamma>0$ such that

$$
\|T(x)-T(y)\| \leq \gamma\|x-y\|, \quad \forall x, y \in \mathcal{H} .
$$

In the next definitions, several generalizations of the nonexpansive mappings which have been introduced by various authors in recent years are stated.

Definition 2.12. A nonlinear mapping $T: \mathcal{H} \rightarrow \mathcal{H}$ is said to be:

(a) nonexpansive if

$$
\|T x-T y\| \leq\|x-y\|, \quad \forall x, y \in \mathcal{H} ;
$$

(b) L-Lipschitzian if there exists a constant $L>0$ such that

$$
\|T x-T y\| \leq L\|x-y\|, \quad \forall x, y \in \mathcal{H}
$$

(c) generalized Lipschitzian if there exists a constant $L>0$ such that

$$
\|T x-T y\| \leq L(\|x-y\|+1), \quad \forall x, y \in \mathcal{H} ;
$$

(d) generalized (L, M)-Lipschitzian [53] if there exist two constants $L, M>0$ such that

$$
\|T x-T y\| \leq L(\|x-y\|+M), \quad \forall x, y \in \mathcal{H}
$$

(e) asymptotically nonexpansive [54] if there exists a sequence $\left\{k_{n}\right\} \subseteq[1, \infty)$ with $\lim _{n \rightarrow \infty} k_{n}=1$ such that, for each $n \in \mathbb{N}$,

$$
\left\|T^{n} x-T^{n} y\right\| \leq k_{n}\|x-y\|, \quad \forall x, y \in \mathcal{H} ;
$$

(f) pointwise asymptotically nonexpansive [55] if, for each integer $n \in \mathbb{N}$,

$$
\left\|T^{n} x-T^{n} y\right\| \leq \alpha_{n}(x)\|x-y\|, \quad \forall x, y \in \mathcal{H},
$$

where $\alpha_{n} \rightarrow 1$ pointwise on $X$; 
(g) uniformly L-Lipschitzian if there exists a constant $L>0$ such that, for each $n \in$ $\mathbb{N}$,

$$
\left\|T^{n} x-T^{n} y\right\| \leq L\|x-y\|, \quad \forall x, y \in \mathcal{H} .
$$

Definition 2.13. [53] A nonlinear mapping $T: \mathcal{H} \rightarrow \mathcal{H}$ is said to be:

(a) nearly Lipschitzian with respect to the sequence $\left\{a_{n}\right\}$ if for each $n \in \mathbb{N}$, there exists a constant $k_{n}>0$ such that

$$
\left\|T^{n} x-T^{n} y\right\| \leq k_{n}\left(\|x-y\|+a_{n}\right), \quad \forall x, y \in \mathcal{H},
$$

where $\left\{a_{n}\right\}$ is a fix sequence in $[0, \infty)$ with $a_{n} \rightarrow 0$ as $n \rightarrow \infty$.

The infimum of constants $k_{n}$ in (2.1) is called nearly Lipschitz constant, which is denoted by $\eta\left(T^{n}\right)$. Notice that

$$
\eta\left(T^{n}\right)=\sup \left\{\frac{\left\|T^{n} x-T^{n} y\right\|}{\|x-y\|+a_{n}}: x, y \in \mathcal{H}, x \neq y\right\} .
$$

A nearly Lipschitzian mapping $T$ with the sequence $\left\{\left(a_{n}, \eta\left(T^{n}\right)\right)\right\}$ is said to be:

(b) nearly nonexpansive if $\eta\left(T^{n}\right)=1$ for all $n \in \mathbb{N}$, that is,

$$
\left\|T^{n} x-T^{n} y\right\| \leq\|x-y\|+a_{n}, \quad \forall x, y \in \mathcal{H} ;
$$

(c) nearly asymptotically nonexpansive if $\eta\left(T^{n}\right) \leq 1$ for all $n \in \mathbb{N}$ and $\lim _{n \rightarrow \infty} \eta\left(T^{n}\right)=1$, in other words, $k_{n} \geq 1$ for all $n \in \mathbb{N}$ with $\lim _{n \rightarrow \infty} k_{n}=1$;

(d) nearly uniformly L-Lipschitzian if $\eta\left(T^{n}\right) \leq L$ for all $n \in \mathbb{N}$, in other words, $k_{n}=$ $L$ for all $n \in \mathbb{N}$.

Remark 2.14. It should be pointed that

(1) Every nonexpansive mapping is a asymptotically nonexpansive mapping and every asymptotically non-expansive mapping is a pointwise asymptotically nonexpansive mapping. Also, the class of Lipschitzian mappings properly includes the class of pointwise asymptotically nonexpansive mappings.

(2) It is obvious that every Lipschitzian mapping is a generalized Lipschitzian mapping. Furthermore, every mapping with a bounded range is a generalized Lipschitzian mapping. It is easy to see that the class of generalized $(L, M)$-Lipschitzian mappings is more general that the class of generalized Lipschitzian mappings.

(3) Clearly, the class of nearly uniformly $L$-Lipschitzian mappings properly includes the class of generalized $(L, M)$-Lipschitzian mappings and that of uniformly $L$ Lipschitzian mappings. Note that every nearly asymptotically nonexpansive mapping is nearly uniformly $L$-Lipschitzian. 
Now, we present some new examples to investigate relations between these mappings.

Example 2.15. Let $\mathcal{H}=\mathbb{R}$ and define a mapping $T: \mathcal{H} \rightarrow \mathcal{H}$ as follow:

$$
T(x)= \begin{cases}\frac{1}{\gamma} & x \in[0, \gamma] \\ 0 & x \in(-\infty, 0) \cup(\gamma, \infty),\end{cases}
$$

where $\gamma>1$ is a constant real number. Evidently, the mapping $T$ is discontinuous at the points $x=0, \gamma$. Since every Lipschitzian mapping is continuous, it follows that $T$ is not Lipschitzian. For each $n \in \mathbb{N}$, take $a_{n}=\frac{1}{\gamma^{n}}$. Then,

$$
|T x-T y| \leq|x-y|+\frac{1}{\gamma}=|x-y|+a_{1}, \quad \forall x, y \in \mathbb{R} .
$$

Since $T^{n} z=\frac{1}{\gamma}$ for all $z \in \mathbb{R}$ and $n \geq 2$, it follows that for all $x, y \in \mathbb{R}$ and $n \geq 2$,

$$
\left|T^{n} x-T^{n} y\right| \leq|x-y|+\frac{1}{\gamma^{n}}=|x-y|+a_{n} .
$$

Hence $T$ is a nearly nonexpansive mapping with respect to the sequence $\left\{a_{n}\right\}=\left\{\frac{1}{\gamma^{n}}\right\}$.

The following example shows that the nearly uniformly $L$-Lipschitzian mappings are not necessarily continuous.

Example 2.16. Let $\mathcal{H}=[0, b]$, where $b \in(0,1]$ is an arbitrary constant real number, and the self-mapping $T$ of $\mathcal{H}$ be defined as below:

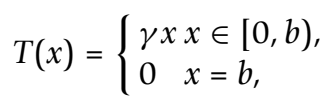

where $\gamma \in(0,1)$ is also an arbitrary constant real number. It is plain that the mapping $T$ is discontinuous in the point $b$. Hence $T$ is not a Lipschitzian mapping. For each $n \in \mathbb{N}$, take $a_{n}=\gamma^{n-1}$. Then, for all $n \in \mathbb{N}$ and $x, y \in[0, b)$, we have

$$
\begin{aligned}
\left|T^{n} x-T^{n} y\right| & =\left|\gamma^{n} x-\gamma^{n} y\right|=\gamma^{n}|x-\gamma| \leq \gamma^{n}|x-\gamma|+\gamma^{n} \\
& \leq \gamma|x-\gamma|+\gamma^{n}=\gamma\left(|x-\gamma|+a_{n}\right) .
\end{aligned}
$$

If $x \in[0, b)$ and $y=b$, then, for each $n \in \mathbb{N}$, we have $T^{n} x=\gamma^{n} x$ and $T^{n} y=0$. Since 0 $<|x-y| \leq b \leq 1$, it follows that, for all $n \in \mathrm{N}$,

$$
\begin{aligned}
\left|T^{n} x-T^{n} y\right|= & \left|\gamma^{n} x-0\right|=\gamma^{n} x \leq \gamma^{n} b \leq \gamma^{n}<\gamma^{n}|x-\gamma|+\gamma^{n} \\
& \leq \gamma|x-\gamma|+\gamma^{n}=\gamma\left(|x-\gamma|+a_{n}\right) .
\end{aligned}
$$

Hence $T$ is a nearly uniformly $\gamma$-Lipschitzian mapping with respect to the sequence $\left\{a_{n}\right\}=\left\{\gamma^{n-1}\right\}$.

Obviously, every nearly nonexpansive mapping is a nearly uniformly Lipschitzian mapping. In the following example, we show that the class of nearly uniformly Lipschitzian mappings properly includes the class of nearly nonexpansive mappings. 
Example 2.17. Let $\mathcal{H}=\mathbb{R}$ and the self-mapping $T$ of $\mathcal{H}$ be defined as follow:

$$
T(x)=\left\{\begin{array}{l}
\frac{1}{2} x \in[0,1) \cup\{2\} \\
2 x=1 \\
0 x \in(-\infty, 0) \cup(1,2) \cup(2,+\infty) .
\end{array}\right.
$$

Evidently, the mapping $T$ is discontinuous in the points $x=0,1,2$. Hence $T$ is not a Lipschitzian mapping. Take for each $n \in \mathrm{N}, a_{n}=\frac{1}{2^{n}}$. Then $T$ is not a nearly nonexpansive mapping with respect to the sequence $\left\{\frac{1}{2^{n}}\right\}$, because taking $x=1$ and $y=\frac{1}{2}$, we have $T x=2, T y=\frac{1}{2}$ and

$$
|T x-T y|>|x-y|+\frac{1}{2}=|x-y|+a_{1}
$$

However,

$$
|T x-T y| \leq 4\left(|x-y|+\frac{1}{2}\right)=4\left(|x-y|+a_{1}\right), \quad \forall x, y \in \mathbb{R}
$$

and for all $n \geq 2$,

$$
\left|T^{n} x-T^{n} y\right| \leq 4\left(|x-y|+\frac{1}{2^{n}}\right)=4\left(|x-y|+a_{n}\right), \quad \forall x, y \in \mathbb{R},
$$

since $T^{n} z=\frac{1}{2}$ for all $z \in \mathbb{R}$ and $n \geq 2$. Hence, for each $L \geq 4, T$ is a nearly uniformly $L$-Lipschitzian mapping with respect to the sequence $\left\{\frac{1}{2^{n}}\right\}$.

It is clear that every uniformly $L$-Lipschitzian mapping is a nearly uniformly $L$ Lipschitzian mapping. In the next example, we show that the class nearly uniformly $L$ Lipschitzian mappings properly includes the class of uniformly $L$-Lipschitzian mappings.

Example 2.18. Let $\mathcal{H}=\mathbb{R}$ and let the self-mapping $T$ of $\mathcal{H}$ be defined the same as in Example 2.17. Then $T$ is not a uniformly 4-Lipschitzian mapping. In fact, if $x=1$ and $y \in\left(1, \frac{3}{2}\right)$, then we have $|T x-T y|>4|x-y|$ because $0<|x-y|<\frac{1}{2}$. But, in view of Example 2.17, $T$ is a nearly uniformly 4-Lipschitzian mapping.

The following example shows that the class of generalized Lipschitzian mappings properly includes the class of Lipschitzian mappings and that of mappings with bounded range.

Example 2.19. [26] Let $\mathcal{H}=\mathbb{R}$ and a mapping $T: \mathcal{H} \rightarrow \mathcal{H}$ be defined by

$$
T(x)= \begin{cases}x-1 & x \in(-\infty,-1), \\ x-\sqrt{1-(x+1)^{2}} & x \in[-1,0) \\ x+\sqrt{1-(x-1)^{2}} & x \in[0,1] \\ x+1 & x \in(1, \infty) .\end{cases}
$$

Then $T$ is a generalized Lipschitzian mapping which is not Lipschitzian and whose range is not bounded. 


\section{Extended general regularized nonconvex variational inequality}

In this section, we introduce a new problem of extended general nonlinear regularized nonconvex variational inequality and some special cases of the problem in Hilbert spaces and investigate their relations.

Let $T, f, g: \mathcal{H} \rightarrow \mathcal{H}$ be three nonlinear single-valued operators such that $K_{r} \subseteq f(\mathcal{H})$. We consider the problem of finding $u \in \mathcal{H}$ such that $g(u) \in K_{r}$ and

$$
\langle\rho T(u)+g(u)-f(u), f(v)-g(u)\rangle+\frac{1}{2 r}\|f(v)-g(u)\|^{2} \geq 0, \quad \forall v \in \mathcal{H}: f(v) \in K_{r},
$$

where $\rho>0$ is a constant. The problem (3.1) is called the extended general nonlinear regularized nonconvex variational inequality involving three different nonlinear operators (EGNRNVID).

Proposition 3.1. If $K_{r}$ is a uniformly prox-regular set, then the problem (3.1) is equivalent to that of finding $u \in \mathcal{H}$ such that $g(u) \in K_{r}$ and

$$
0 \in \rho T(u)+g(u)-f(u)+N_{K_{r}}^{P}(g(u))
$$

where $N_{K_{r}}^{P}(s)$ denotes the P-normal cone of $K_{r}$ at $s$ in the sense of nonconvex analysis.

Proof. Let $u \in \mathcal{H}$ with $g(u) \in K_{r}$ be a solution of the problem (3.1). If $\rho T(u)+g(u)$ $f(u)=0$, because the vector zero always belongs to any normal cone, we have $0 \in \rho T(u)+g(u)-f(u)+N_{K_{r}}^{P}(g(u))$. If $\rho T(u)+g(u)-f(u) \neq 0$, then for all $v \in \mathcal{H}$ with $f(v) \in K_{r}$, one has

$$
\langle-(\rho T(u)+g(u)-f(u)), f(v)-g(u)\rangle \leq \frac{1}{2 r}\|f(v)-g(u)\|^{2} .
$$

Now, by using Lemma 2.3 conclude that $-(\rho T(u)+g(u)-f(u)) \in N_{K_{r}}^{P}(g(u))$ and so

$$
0 \in \rho T(u)+g(u)-f(u)+N_{K_{r}}^{P}(g(u)) .
$$

Conversely, if $u \in \mathcal{H}$ with $g(u) \in K_{r}$ is a solution of the problem (3.2), then Definition 2.8 guarantees that $u \in \mathcal{H}$ with $g(u) \in K_{r}$ is a solution of the problem (3.1). This completes the proof.

The problem (3.2) is called the extended general nonconvex variational inclusion associated with EGNRNVID problem.

Some special cases of the problem (3.1) are as follows:

(1) If $g \equiv I$ (: the identity operator), then the problem (3.1) collapses to the following problem: Find $u \in K_{r}$ such that

$$
\langle\rho T(u)+u-f(u), f(v)-u\rangle+\frac{1}{2 r}\|f(v)-u\|^{2} \geq 0, \quad \forall v \in \mathcal{H}: f(v) \in K_{r},
$$

which is a new problem of general nonlinear regularized nonconvex variational inequality involving two nonlinear operators (GNRNVID).

(2) If $f=g$, then the problem (3.1) reduces to the following problem: Find $u \in \mathcal{H}$ such that $g(u) \in K_{r}$ and 


$$
\langle\rho T(u), g(v)-g(u)\rangle+\frac{1}{2 r}\|g(v)-g(u)\|^{2} \geq 0, \quad \forall v \in \mathcal{H}: g(v) \in K_{r},
$$

which is also a new problem of general nonlinear regularized nonconvex variational inequality involving two nonlinear operators (GNRNVID).

(3) If $g \equiv I$, then the problem (3.4) collapses to the following problem: Find $u \in K_{r}$ such that

$$
\langle\rho T(u), v-u\rangle+\frac{1}{2 r}\|v-u\|^{2} \geq 0, \quad \forall v \in K_{r},
$$

which is a new problem of nonlinear regularized nonconvex variational inequality (NRNVI).

(4) If $r=\infty$, i.e., $K_{r}=K$, the convex set in $\mathcal{H}$, then the problem (3.1) changes into that of finding $u \in \mathcal{H}$ such that $g(u) \in K$ and

$$
\langle\rho T(u)+g(u)-f(u), f(v)-g(u)\rangle \geq 0, \quad \forall v \in \mathcal{H}: f(v) \in K .
$$

The inequality of type (3.6) is introduced and studied by Noor [33,39].

(5) If $r=\infty$, then the problem (3.3) is equivalent to the problem: Find $u L K$ such that

$$
\langle\rho T(u)+u-f(u), f(v)-u\rangle \geq 0, \quad \forall v \in \mathcal{H}: f(v) \in K .
$$

The problem (3.7) is introduced and studied by Noor [34].

(6) If $r=\infty$, then the problem (3.4) reduces to the following problem: Find $u \in \mathcal{H}$ such that $g(u) \in K$ and

$$
\langle T(u), g(v)-g(u)\rangle \geq 0, \quad \forall v \in \mathcal{H}: g(v) \in K,
$$

which is known as the general nonlinear variational inequality introduced and studied by Noor [37] in 1988.

(7) If $r=\infty$, then the problem (3.5) changes into the problem: Find $u \in K$ such that

$$
\langle T u, v-u\rangle \geq 0, \quad \forall v \in K
$$

The inequality of type (3.9) is called variational inequality, which was introduced and studied by Stampacchia [1] in 1964.

Now, we prove the existence and uniqueness theorem for solution of the problem of extended general nonlinear regularized nonconvex variational inequality (3.1). For this end, we need to the following lemma in which by using the projection operator technique, we verify the equivalence between the problem (3.1) and the fixed point problem.

Lemma 3.2. Let $T, f, g$ and $\rho>0$ be the same as in the problem (3.1). Then $u \in \mathcal{H}$ with $g(u) \in K_{r}$ is a solution of the problem (3.1) if and only if 


$$
g(u)=P_{K_{r}}(f(u)-\rho T(u))
$$

where $P_{K_{r}}$ is the projection of $\mathcal{H}$ onto $K_{r}$.

Proof. Let $u \in \mathcal{H}$ with $g(u) \in K_{r}$ be a solution of the problem (3.1). Then, by using Proposition 3.1, we have

$$
\begin{aligned}
& 0 \in \rho T(u)+g(u)-f(u)+N_{K_{r}}^{P}(g(u)) \\
& \Leftrightarrow \quad f(u)-\rho T(u) \in g(u)+N_{K_{r}}^{P}(g(u)) \\
& \Leftrightarrow \quad f(u)-\rho T(u) \in\left(I+N_{K_{r}}^{P}\right)(g(u)) \\
& \Leftrightarrow \quad g(u)=P_{K_{r}}(f(u)-\rho T(u)),
\end{aligned}
$$

where $I$ is identity operator and we have used the well-known fact that $P_{K_{r}}=\left(I+N_{K_{r}}^{P}\right)^{-1}$.

Theorem 3.3. Let T, f, $g$ and $\rho$ be the same as in the problem (3.1) such that

(a) $T$ is $\kappa$-strongly monotone with respect to $f$ and $\sigma$-Lipschitz continuous;

(b) $g$ is $\tau$-strongly monotone and i-Lipschitz continuous;

(c) $f$ is w-Lipschitz continuous.

If the constant $\rho>0$ satisfies the following condition:

$$
\left\{\begin{array}{l}
\left|\rho-\frac{\kappa}{\sigma^{2}}\right|<\frac{\sqrt{r^{2} \kappa^{2}-\sigma^{2}\left(r^{2} \varpi^{2}-\left(r-r^{\prime}\right)^{2}(1-\mu)^{2}\right)}}{r \sigma^{2}}, \\
r \kappa>\sigma \sqrt{r^{2} \varpi^{2}-\left(r-r^{\prime}\right)^{2}(1-\mu)^{2}}, \\
r \varpi>\left(r-r^{\prime}\right)(1-\mu), \quad \mu=\sqrt{1-\left(2 \tau-\iota^{2}\right)}<1, \\
2 \tau<1+\iota^{2},
\end{array}\right.
$$

where $r^{\prime} \in(0, r)$, then the problem (3.1) admits a unique solution.

Proof. Define the mapping $\phi: \mathcal{H} \rightarrow \mathcal{H}$ by

$$
\phi(x)=x-g(x)+P_{K_{r}}(f(x)-\rho T(x)), \quad \forall x \in \mathcal{H}: g(x) \in K_{r} .
$$

Now, we establish that $\varphi$ is a contraction mapping. Let $x, \hat{x} \in \mathcal{H}$ with $g(x), g(\hat{x}) \in K_{r}$ be given. It follows from Proposition 2.10 that

$$
\begin{aligned}
\|\phi(x)-\phi(\hat{x})\| & \leq\|x-\hat{x}-(g(x)-g(\hat{x}))\|+\left\|P_{K_{r}}(f(x)-\rho T(x))-P_{K_{r}}(f(\hat{x})-\rho T(\hat{x}))\right\| \\
& \leq\|x-\hat{x}-(g(x)-g(\hat{x}))\|+\frac{r}{r-r^{\prime}}\|f(x)-f(\hat{x})-\rho(T(x)-T(\hat{x}))\| .
\end{aligned}
$$

By using $\tau$-strongly monotonicity and $\iota$-Lipschitzian continuity of $g$, we have

$$
\begin{aligned}
\|x-\hat{x}-(g(x)-g(\hat{x}))\|^{2} & =\|x-\hat{x}\|^{2}-2\langle g(x)-g(\hat{x}), x-\hat{x}\rangle+\|g(x)-g(\hat{x})\|^{2} \\
& \leq\left(1-2 \tau+\iota^{2}\right)\|x-\hat{x}\|^{2} .
\end{aligned}
$$

Since $T$ is $\kappa$-strongly monotone with respect to $f$ and $\sigma$-Lipschitzian continuous, and $f$ is $\varpi$-Lipschitzian continuous, we gain

$$
\begin{aligned}
& \|f(x)-f(\hat{x})-\rho(T(x)-T(\hat{x}))\|^{2} \\
& =\|f(x)-f(\hat{x})\|^{2}-2 \rho\langle T(x)-T(\hat{x}), f(x)-f(\hat{x})\rangle+\rho^{2}\|T(x)-T(\hat{x})\|^{2} \\
& \leq\left(\varpi^{2}-2 \rho \kappa+\rho^{2} \sigma^{2}\right)\|x-\hat{x}\|^{2} .
\end{aligned}
$$


Substituting (3.14) and (3.15) for (3.13), we obtain

$$
\|\phi(x)-\phi(\hat{x})\| \leq \gamma\|x-\hat{x}\|
$$

where

$$
\gamma=\sqrt{1-2 \tau+\iota^{2}}+\frac{r}{r-r^{\prime}} \sqrt{\varpi^{2}-2 \rho \kappa+\rho^{2} \sigma^{2}} .
$$

In view of the condition (3.11), we note that $0 \leq \gamma<1$ and so from (3.16) conclude that the mapping $\varphi$ is contraction. According to Banach fixed point theorem, $\varphi$ has a unique fixed point in $\mathcal{H}$, that is, there exists a unique point $u \in \mathcal{H}$ with $g(u) \in K_{r}$ such that $\varphi(u)=u$. It follows from (3.12) that $g(u)=P_{K_{r}}(f(u)-\rho T(u))$. Now, Lemma 3.2 guarantees that $u \in \mathcal{H}$ with $g(u) \in K_{r}$ is a solution of the problem (3.1). This completes the proof.

As in the proof of Theorem 3.3, one can prove the existence and uniqueness theorem for solution of the problems (3.3)-(3.5) and we omit their proofs.

Theorem 3.4. Assume that $T, f$ and $\rho$ are the same as in the problem (3.3) such that

(a) $T$ is $\kappa$-strongly monotone with respect to $f$ and $\sigma$-Lipschitz continuous;

(b) $f$ is $\boldsymbol{\omega}$-Lipschitz continuous.

If the constant $\rho>0$ satisfies the following condition:

$$
\left\{\begin{array}{l}
\left|\rho-\frac{\kappa}{\sigma^{2}}\right|<\frac{\sqrt{r^{2} \kappa^{2}-\sigma^{2}\left(r^{2} \varpi^{2}-\left(r-r^{\prime}\right)^{2}\right)}}{r \sigma^{2}} \\
r \kappa>\sigma \sqrt{r^{2} \varpi^{2}-\left(r-r^{\prime}\right)^{2}} \\
r \varpi>\left(r-r^{\prime}\right)
\end{array}\right.
$$

where $r^{\prime} \in(0, r)$, then the problem (3.3) admits a unique solution.

Theorem 3.5. Let T, $g$ and $\rho$ be the same as in the problem (3.4) such that

(a) $T$ is $\kappa$-strongly monotone with respect to $f$ and $\sigma$-Lipschitz continuous;

(b) $g$ is $\tau$-strongly monotone and l-Lipschitz continuous.

If the constant $\rho>0$ satisfies the following condition:

$$
\left\{\begin{array}{l}
\left|\rho-\frac{\kappa}{\sigma^{2}}\right|<\frac{\sqrt{r^{2} \kappa^{2}-\sigma^{2}\left(r^{2} \iota^{2}-\left(r-r^{\prime}\right)^{2}(1-\mu)^{2}\right)}}{r \sigma^{2}} \\
r \kappa>\sigma \sqrt{r^{2} \iota^{2}-\left(r-r^{\prime}\right)^{2}(1-\mu)^{2}} \\
r \iota>\left(r-r^{\prime}\right)(1-\mu), \quad \mu=\sqrt{1-\left(2 \tau-\iota^{2}\right)}<1, \\
2 \tau<1+\iota^{2}
\end{array}\right.
$$

where $r^{\prime} \in(0, r)$, then the problem (3.4) admits a unique solution.

Theorem 3.6. Suppose that $T$ and $\rho$ are the same as in the problem (3.5) such that $T$ is $\kappa$-strongly monotone and $\sigma$-Lipschitz continuous. If the constant $\rho>0$ satisfies the following condition:

$$
\left|\rho-\frac{\kappa}{\sigma^{2}}\right|<\frac{\sqrt{r^{2} \kappa^{2}-\sigma^{2} r^{\prime}\left(r^{\prime}-2 r\right)}}{r \sigma^{2}}
$$


where $r^{\prime} \in(0, r)$, then the problem (3.5) admits a unique solution.

\section{Nearly uniformly Lipschitzian mappings and finite step projection iterative algorithms}

In this section, applying a nearly uniformly Lipschitzian mapping $S$ and by using the fixed point formulation (3.10), we suggest and analyze some new $p$-step projection iterative algorithms with mixed errors for finding an element of set of the fixed points of nearly uniformly Lipschitzian mapping $S$ which is unique solution of the problem of extended general nonlinear regularized nonconvex variational inequality (3.1).

Let $S: K_{r} \rightarrow K_{r}$ be a nearly uniformly Lipschitzian mapping. We denote the set of all the fixed points of $S$ by $\operatorname{Fix}(S)$ and the set of all the solutions of the problem (3.1) by $\operatorname{EGNRNVID}\left(K_{r}, T, f, g\right)$. We now characterize the problem. If $u \in \operatorname{Fix}(S) \cap$ EGNRN$\operatorname{VID}\left(K_{r}, T, f, g\right)$, then it follows from Lemma 3.2 that, for each $n \geq 0$,

$$
u=S^{n} u=u-g(u)+P_{K_{r}}(f(u)-\rho T(u))=S^{n}\left\{u-g(u)+P_{K_{r}}(f(u)-\rho T(u))\right\} .
$$

The fixed point formulation (4.1) enables us to define the following $p$-step projection iterative algorithms with mixed errors for finding a common element of two different sets of solutions of the fixed points of the nearly uniformly Lipschitzian mappings and the extended general nonlinear regularized nonconvex variational inequalities (3.1).

Algorithm 4.1. Let $T, f, g$ and $\rho$ be the same as in the problem (3.1). For arbitrary chosen initial point $x_{0} \in K_{r}$, compute the iterative sequence $\left\{x_{n}\right\}_{n=0}^{\infty}$ by the iterative process

$$
\begin{aligned}
x_{n+1} & =\left(1-\alpha_{n, 1}-\beta_{n, 1}\right) x_{n}+\alpha_{n, 1}\left(S^{n} \Psi\left(y_{n, 1}\right)+e_{n, 1}\right)+\beta_{n, 1} l_{n, 1}+r_{n, 1} \\
y_{n, i} & =\left(1-\alpha_{n, i+1}-\beta_{n, i+1}\right) x_{n}+\alpha_{n, i+1}\left(S^{n} \Psi\left(y_{n, i+1}\right)+e_{n, i+1}\right)+\beta_{n, i+1} l_{n, i+1}+r_{n, i+1}, \\
\quad \ldots & \\
y_{n, p-1} & =\left(1-\alpha_{n, p}-\beta_{n, p}\right) x_{n}+\alpha_{n, p}\left(S^{n} \Psi\left(x_{n}\right)+e_{n, p}\right)+\beta_{n, p} l_{n, p}+r_{n, p} \\
i & =1,2, \ldots, p-2
\end{aligned}
$$

where

$$
\left\{\begin{array}{l}
\Psi\left(y_{n, i}\right)=y_{n, i}-g\left(y_{n, i}\right)+P_{K_{r}}\left(f\left(y_{n, i}\right)-\rho T\left(y_{n, i}\right)\right) \\
\Psi\left(x_{n}\right)=x_{n}-g\left(x_{n}\right)+P_{K_{r}}\left(f\left(x_{n}\right)-\rho T\left(x_{n}\right)\right) \\
i=1,2, \ldots, p-2
\end{array}\right.
$$

$S: K_{r} \rightarrow K_{r}$ is a nearly uniformly Lipschitzian mapping, $\left\{\alpha_{n, i}\right\}_{n=0}^{\infty}$, $\left\{\beta_{n, i}\right\}_{n=0}^{\infty}(i=1,2, \ldots, p)$ are $2 p$ sequences in interval $[0,1]$ such that $\sum_{n=0}^{\infty} \beta_{n, i}<\infty, \sum_{n=0}^{\infty} \beta_{n, i}<\infty$, and $\left\{e_{n, i}\right\}_{n=0}^{\infty},\left\{l_{n, i}\right\}_{n=0}^{\infty},\left\{r_{n, i}\right\}_{n=0}^{\infty}(i=1,2, \ldots, p)$ are $3 p$ sequences in $\mathcal{H}$ to take into account a possible inexact computation of the resolvent operator point satisfying the following conditions: $\left\{l_{n, i}\right\}_{n=0}^{\infty}(i=1,2, \ldots, p)$ are $p$ bounded sequences in $\mathcal{H}$ and $\sum_{n=0}^{\infty} \beta_{n, i}<\infty,\left\{r_{n, i}\right\}_{n=0}^{\infty}$ are $2 p$ sequences in $\mathcal{H}$ such that 


$$
\begin{cases}e_{n, i}=e^{\prime}{ }_{n, i}+e^{\prime \prime}{ }_{n, i}, & n \geq 0, \quad i=1,2, \ldots, p \\ \lim _{n \rightarrow \infty}\left\|e_{n, i}^{\prime}\right\|=0, & i=1,2, \ldots, p, \\ \sum_{n=0}^{\infty}\left\|e^{\prime \prime}{ }_{n, i}\right\|<\infty, & \sum_{n=0}^{\infty}\left\|r_{n, i}\right\|<\infty, \quad i=1,2, \ldots, p .\end{cases}
$$

Algorithm 4.2. Assume that $T, f$ and $\rho$ are the same as in the problem (3.3). For arbitrary chosen initial point $x_{0} \in K_{r}$, compute the iterative sequence $\left\{x_{n}\right\}_{n=0}^{\infty}$ by the iterative process

$$
\left\{\begin{array}{l}
x_{n+1}=\left(1-\alpha_{n, 1}-\beta_{n, 1}\right) x_{n}+\alpha_{n, 1}\left(S^{n} P_{K_{r}}\left(f\left(y_{n, 1}\right)-\rho T\left(y_{n, 1}\right)\right)+e_{n, 1}\right)+\beta_{n, 1} l_{n, 1}+r_{n, 1} \\
y_{n, i}=\left(1-\alpha_{n, i+1}-\beta_{n, i+1}\right) x_{n}+\alpha_{n, i+1}\left(S^{n} P_{K_{r}}\left(f\left(y_{n, i+1}\right)-\rho T\left(y_{n, i+1}\right)\right)+e_{n, i+1}\right)+\beta_{n, i+1} l_{n, i+1}+r_{n, i+1} \\
\cdots \\
y_{n, p-1}=\left(1-\alpha_{n, p}-\beta_{n, p}\right) x_{n}+\alpha_{n, p}\left(S^{n} P_{K_{r}}\left(f\left(x_{n}\right)-\rho T\left(x_{n}\right)\right)+e_{n, p}\right)+\beta_{n, p} l_{n, p}+r_{n, p} \\
i=1,2, \ldots, p-2
\end{array}\right.
$$

where $S,\left\{\alpha_{n, i}\right\}_{n=0}^{\infty},\left\{\beta_{n, i}\right\}_{n=0}^{\infty},\left\{e_{n, i}\right\}_{n=0}^{\infty},\left\{l_{n, i}\right\}_{n=0}^{\infty},\left\{r_{n, i}\right\}_{n=0}^{\infty}(i=1,2, \ldots, p)$ are the same as in Algorithm 4.1.

Algorithm 4.3. Let $T, g$ and $\rho$ be the same as in the problem (3.4). For arbitrary chosen initial point $x_{0} \in K_{r}$, compute the iterative sequence $\left\{x_{n}\right\}_{n=0}^{\infty}$ as follows:

$$
\left\{\begin{array}{l}
x_{n+1}=\left(1-\alpha_{n, 1}-\beta_{n, 1}\right) x_{n}+\alpha_{n, 1}\left(S^{n} \Phi\left(y_{n, 1}\right)+e_{n, 1}\right)+\beta_{n, 1} l_{n, 1}+r_{n, 1}, \\
y_{n, i}=\left(1-\alpha_{n, i+1}-\beta_{n, i+1}\right) x_{n}+\alpha_{n, i+1}\left(S^{n} \Phi\left(y_{n, i+1}\right)+e_{n, i+1}\right)+\beta_{n, i+1} l_{n, i+1}+r_{n, i+1}, \\
\cdots \\
y_{n, p-1}=\left(1-\alpha_{n, p}-\beta_{n, p}\right) x_{n}+\alpha_{n, p}\left(S^{n} \Phi\left(x_{n}\right)+e_{n, p}\right)+\beta_{n, p} l_{n, p}+r_{n, p}, \\
i=1,2, \ldots, p-2,
\end{array}\right.
$$

where

$$
\left\{\begin{array}{l}
\Phi\left(y_{n, i}\right)=y_{n, i}-g\left(y_{n, i}\right)+P_{K_{r}}\left(g\left(y_{n, i}\right)-\rho T\left(y_{n, i}\right)\right) \\
\Phi\left(x_{n}\right)=x_{n}-g\left(x_{n}\right)+P_{K_{r}}\left(g\left(x_{n}\right)-\rho T\left(x_{n}\right)\right) \\
i=1,2, \ldots, p-2
\end{array}\right.
$$

and $S,\left\{\alpha_{n, i}\right\}_{n=0}^{\infty},\left\{\beta_{n, i}\right\}_{n=0}^{\infty},\left\{e_{n, i}\right\}_{n=0}^{\infty},\left\{l_{n, i}\right\}_{n=0}^{\infty},\left\{r_{n, i}\right\}_{n=0}^{\infty}(i=1,2, \ldots, p)$ are the same as in Algorithm 4.1.

Algorithm 4.4. Let $T$ and $\rho$ be the same as in the problem (3.5). For arbitrary chosen initial point $x_{0} \in K_{r}$, compute the iterative sequence $\left\{x_{n}\right\}_{n=0}^{\infty}$ by using

$$
\left\{\begin{array}{l}
x_{n+1}=\left(1-\alpha_{n, 1}-\beta_{n, 1}\right) x_{n}+\alpha_{n, 1}\left(S^{n} P_{K_{r}}\left(y_{n, 1}-\rho T\left(y_{n, 1}\right)\right)+e_{n, 1}\right)+\beta_{n, 1} l_{n, 1}+r_{n, 1}, \\
y_{n, i}=\left(1-\alpha_{n, i+1}-\beta_{n, i+1}\right) x_{n}+\alpha_{n, i+1}\left(S^{n} P_{K_{r}}\left(y_{n, i+1}-\rho T\left(y_{n, i+1}\right)\right)+e_{n, i+1}\right)+\beta_{n, i+1} l_{n, i+1}+r_{n, i+1}, \\
\cdots \\
y_{n, p-1}=\left(1-\alpha_{n, p}-\beta_{n, p}\right) x_{n}+\alpha_{n, p}\left(S^{n} P_{K_{r}}\left(x_{n}-\rho T\left(x_{n}\right)\right)+e_{n, p}\right)+\beta_{n, p} l_{n, p}+r_{n, p} \\
i=1,2, \ldots, p-2
\end{array}\right.
$$

where $S,\left\{\alpha_{n, i}\right\}_{n=0}^{\infty},\left\{\beta_{n, i}\right\}_{n=0}^{\infty},\left\{e_{n, i}\right\}_{n=0}^{\infty},\left\{l_{n, i}\right\}_{n=0}^{\infty},\left\{r_{n, i}\right\}_{n=0}^{\infty}(i=1,2, \ldots, p)$ are the same as in Algorithm 4.1.

Remark 4.5. It should be pointed out that

(1) If $e_{n, i}=r_{n, i}=0$, for all $n \geq 0$ and $i=1,2, \ldots, p$, then Algorithms 4.1-4.4 change into the perturbed iterative process with mean errors.

(2) When $e_{n, i}=l_{n, i}=r_{n, i}=0$, for all $n \geq 0$ and $i=1,2, \ldots, p$, then Algorithms 4.1-4.4 reduce to the perturbed iterative process without error. 
Remark 4.6. Algorithms 2.1-2.6 in [38] and Algorithm 2.1 in [44] are special cases of Algorithms 4.1-4.4. In brief, for a suitable and appropriate choice of the operators $T, f$, $g$, the constant $\rho$, and the sequences $\left\{\alpha_{n, i}\right\}_{n=0}^{\infty}, \quad\left\{\beta_{n, i}\right\}_{n=0}^{\infty}, \quad\left\{e_{n, i}\right\}_{n=0}^{\infty}, \quad\left\{l_{n, i}\right\}_{n=0}^{\infty}$, $\left\{r_{n, i}\right\}_{n=0}^{\infty}(i=1,2, \ldots, p)$, one can obtain a number of new and previously known iterative schemes for solving the problems (3.1) and (3.3)-(3.5) and related problems. This clearly shows that Algorithms 4.1-4.4 are quite general and unifying.

Now, we discuss the convergence analysis of the suggested iterative Algorithms 4.14.4 under some suitable conditions. For this end, we need to the following lemma:

Lemma 4.7. Let $\left.\left.-a_{n}\right\},-b_{n}\right\}$ and $\left.-c_{n}\right\}$ be three nonnegative real sequences satisfying the following condition: there exists a natural number $n_{0}$ such that

$$
a_{n+1} \leq\left(1-t_{n}\right) a_{n}+b_{n} t_{n}+c_{n}, \quad \forall n \geq n_{0},
$$

where $t_{n} \in[0,1], \sum_{n=0}^{\infty} t_{n}=\infty, \lim _{n \rightarrow \infty} b_{n}=0, \sum_{n=0}^{\infty} c_{n}<\infty$. Then $\lim _{n \rightarrow 0} a_{n}=0$.

Proof. The proof directly follows from Lemma 2 in Liu [32].

Theorem 4.8. Let $T, f, g$ and $\rho$ be the same as in Theorem 3.3 such that the conditions (a)-(c) and (3.11) in Theorem 3.3 hold. Assume that $S: K_{r} \rightarrow K_{r}$ is a nearly uniformly L-Lipschitzian mapping with the sequence $\left\{b_{n}\right\}_{n=0}^{\infty}$ such that $\operatorname{Fix}(S) \cap$ EGNRNVID $\left(K_{r}, T, f, g\right) \neq \varnothing$. Further, let $L \gamma<1$, where $\gamma$ is the same as in (3.17). If there exists a constant $\alpha>0$ such that $\prod_{i=1}^{p} \alpha_{n, i}>\alpha$ for each $n \geq 0$, then the iterative sequence $\left\{x_{n}\right\}_{n=0}^{\infty}$ generated by Algorithm 4.1 converges strongly to the only element of $\operatorname{Fix}(S) \cap$ $\operatorname{EGNRNVID}\left(K_{r}, T, f, g\right)$.

Proof. According to Theorem 3.3, the problem (3.1) has a unique solution $x^{*} \in \mathcal{H}$ with $g\left(x^{*}\right) \in K_{r}$. Hence, in view of Lemma 3.2, $g\left(x^{*}\right)=P_{K_{r}}\left(f\left(x^{*}\right)-\rho T\left(x^{*}\right)\right)$. Since $\operatorname{EGNRNVID}\left(K_{r}, T, f, g\right)$ is a singleton set, it follows from $\operatorname{Fix}(S) \cap \operatorname{EGNRNVID}\left(K_{r}, T, f\right.$, $g) \neq \varnothing$ that $x^{*} \in \operatorname{Fix}(S)$. Accordingly, for each $n \geq 0$ and $i \in\{1,2, \ldots, p\}$, we can write

$$
x^{*}=\left(1-\alpha_{n, i}-\beta_{n, i}\right) x^{*}+\alpha_{n, i} S^{n}\left\{x^{*}-g\left(x^{*}\right)+P_{K_{r}}\left(f\left(x^{*}\right)-\rho T\left(x^{*}\right)\right)\right\}+\beta_{n, i} x^{*},
$$

where the sequences $\left\{\alpha_{n, i}\right\}_{n=0}^{\infty}$ and $\left\{\beta_{n, i}\right\}_{n=0}^{\infty}(i=1,2, \ldots, p)$ are the same as in Algorithm 4.1. Let $\Gamma=\sup _{n \geq 0}\left\{|| l_{n, i}-x^{*}||: i=1,2, . ., p\right\}$. It follows from (4.2), (4.4), Proposition 2.10 and the assumptions that

$$
\begin{aligned}
\left\|x_{n+1}-x^{*}\right\| & \leq\left(1-\alpha_{n, 1}-\beta_{n, 1}\right)\left\|x_{n}-x^{*}\right\|+\alpha_{n, 1} \| S^{n}\left\{y_{n, 1}-g\left(y_{n, 1}\right)+P_{K_{r}}\left(f\left(y_{n, 1}\right)-\rho T\left(y_{n, 1}\right)\right)\right\} \\
& -S^{n}\left\{x^{*}-g\left(x^{*}\right)+P_{K_{r}}\left(f\left(x^{*}\right)-\rho T\left(x^{*}\right)\right)\right\}\left\|+\beta_{n, 1}\right\| l_{n, 1}-x^{*}\left\|+\alpha_{n, 1}\right\| e_{n, 1}\|+\| r_{n, 1} \| \\
\leq & \left(1-\alpha_{n, 1}-\beta_{n, 1}\right)\left\|x_{n}-x^{*}\right\|+\alpha_{n, 1} L\left(\left\|y_{n, 1}-x^{*}-\left(g\left(y_{n, 1}\right)-g\left(x^{*}\right)\right)\right\|\right. \\
& \left.+\frac{r}{r-r^{\prime}}\left\|f\left(y_{n, 1}\right)-f\left(x^{*}\right)-\rho\left(T\left(y_{n, 1}\right)-T\left(x^{*}\right)\right)\right\|+b_{n}\right) \\
& +\alpha_{n, 1}\left\|e_{n, 1}^{\prime}\right\|+\left\|e_{n, 1}^{\prime \prime}\right\|+\left\|r_{n, 1}\right\|+\beta_{n, 1} \Gamma .
\end{aligned}
$$

Since $T$ is $\kappa$-strongly monotone with respect to $f$ and $\sigma$-Lipschitz continuous, $g$ is $\tau$ strongly monotone and $\iota$-Lipschitz continuous, in similar way to the proofs (3.14) and (3.15), we can prove that

$$
\left\|y_{n, 1}-x^{*}-\left(g\left(y_{n, 1}\right)-g\left(x^{*}\right)\right)\right\| \leq \sqrt{1-2 \tau+\iota^{2}}|| y_{n, 1}-x^{*} \|
$$

and

$$
\left\|f\left(y_{n, 1}\right)-f\left(x^{*}\right)-\rho\left(T\left(y_{n, 1}\right)-T\left(x^{*}\right)\right)\right\| \leq \sqrt{\varpi^{2}-2 \rho \kappa+\rho^{2} \sigma^{2}}\left\|y_{n, 1}-x^{*}\right\| .
$$


Substituting (4.6) and (4.7) for (4.5), we obtain

$$
\begin{aligned}
\left\|x_{n+1}-x^{*}\right\| & \leq\left(1-\alpha_{n, 1}-\beta_{n, 1}\right)\left\|x_{n}-x^{*}\right\|+\alpha_{n, 1} L \gamma\left\|y_{n, 1}-x^{*}\right\| \\
& +\alpha_{n, 1} L b_{n}+\alpha_{n, 1}\left\|e_{n, 1}^{\prime}\right\|+\left\|e_{n, 1}^{\prime \prime}\right\|+\left\|r_{n, 1}\right\|+\beta_{n, 1} \Gamma .
\end{aligned}
$$

Like in the proofs of (4.5)-(4.8), we can establish that, for each $i \in\{1,2, \ldots, p-2\}$,

$$
\begin{aligned}
\left\|y_{n, i}-x^{*}\right\| \leq & \left(1-\alpha_{n, i+1}-\beta_{n, i+1}\right)\left\|x_{n}-x^{*}\right\|+\alpha_{n, i+1} L \gamma\left\|y_{n, i+1}-x^{*}\right\| \\
& +\alpha_{n, i+1} L b_{n}+\alpha_{n, i+1}\left\|e_{n, i+1}^{\prime}\right\|+\left\|e_{n, i+1}^{\prime \prime}\right\|+\left\|r_{n, i+1}\right\|+\beta_{n, i+1} \Gamma
\end{aligned}
$$

and

$$
\begin{aligned}
\left\|y_{n, p-1}-x^{*}\right\| & \leq\left(1-\alpha_{n, p}-\beta_{n, p}\right)\left\|x_{n}-x^{*}\right\|+\alpha_{n, p} L \gamma\left\|x_{n}-x^{*}\right\| \\
& +\alpha_{n, p} L b_{n}+\alpha_{n, p}\left\|e_{n, p}^{\prime}\right\|+\left\|e_{n, p}^{\prime \prime}\right\|+\left\|r_{n, p}\right\|+\beta_{n, p} \Gamma .
\end{aligned}
$$

By using (4.9) and (4.10), we get

$$
\begin{aligned}
\left\|y_{n, p-2}-x^{*}\right\| \leq & \left(1-\alpha_{n, p-1}-\beta_{n, p-1}\right)\left\|x_{n}-x^{*}\right\|+\alpha_{n, p-1} L \gamma\left\|y_{n, p-1}-x^{*}\right\| \\
& +\alpha_{n, p-1} L b_{n}+\alpha_{n, p-1}\left\|e_{n, p-1}^{\prime}\right\|+\left\|e_{n, p-1}^{\prime \prime}\right\|+\left\|r_{n, p-1}\right\|+\beta_{n, p-1} \Gamma \\
\leq & \left(1-\alpha_{n, p-1}-\beta_{n, p-1}\right)\left\|x_{n}-x^{*}\right\|+\alpha_{n, p-1} L \gamma\left[\left(1-\alpha_{n, p}-\beta_{n, p}\right)\left\|x_{n}-x^{*}\right\|\right. \\
& \left.+\alpha_{n, p} L \gamma\left\|x_{n}-x^{*}\right\|+\alpha_{n, p} L b_{n}+\alpha_{n, p}\left\|e_{n, p}^{\prime}\right\|+\left\|e_{n, p}^{\prime \prime}\right\|+\left\|r_{n, p}\right\|+\beta_{n, p} \Gamma\right] \\
& +\alpha_{n, p-1} L b_{n}+\alpha_{n, p-1}\left\|e_{n, p-1}^{\prime}\right\|+\left\|e_{n, p-1}^{\prime \prime}\right\|+\left\|r_{n, p-1}\right\|+\beta_{n, p-1} \Gamma \\
= & \left(1-\alpha_{n, p-1}-\beta_{n, p-1}+\alpha_{n, p-1}\left(1-\alpha_{n, p}-\beta_{n, p}\right) L \gamma+\alpha_{n, p-1} \alpha_{n, p} L^{2} \gamma^{2}\right)\left\|x_{n}-x^{*}\right\| \\
& +\left(\alpha_{n, p-1} L+\alpha_{n, p-1} \alpha_{n, p} L^{2} \gamma\right) b_{n}+\alpha_{n, p-1}\left\|e_{n, p-1}^{\prime}\right\|+\alpha_{n, p-1} \alpha_{n, p} L \gamma\left\|e_{n, p}^{\prime}\right\| \\
& +\left\|e_{n, p-1}^{\prime \prime}\right\|+\alpha_{n, p-1} L \gamma\left\|e_{n, p}\right\|+\left\|r_{n, p-1}\right\|+\alpha_{n, p-1} L \gamma\left\|r_{n, p}\right\| \\
& +\left(\beta_{n, p-1}+\alpha_{n, p-1} \beta_{n, p} L \gamma\right) \Gamma .
\end{aligned}
$$

As in the proof of (4.11), applying (4.9) and (4.11), we have

$$
\begin{aligned}
\left\|y_{n, p-3}-x^{*}\right\| & \leq\left(1-\alpha_{n, p-2}-\beta_{n, p-2}+\alpha_{n, p-2}\left(1-\alpha_{n, p-1}-\beta_{n, p-1}\right) L \gamma\right. \\
& \left.+\alpha_{n, p-2} \alpha_{n, p-1}\left(1-\alpha_{n, p}-\beta_{n, p}\right) L^{2} \gamma^{2}+\alpha_{n, p-2} \alpha_{n, p-1} \alpha_{n, p} L^{3} \gamma^{3}\right)\left\|x_{n}-x^{*}\right\| \\
& +\alpha_{n, p-2}\left\|e_{n, p-2}^{\prime}\right\|+\alpha_{n, p-2} \alpha_{n, p-1} L \gamma\left\|e_{n, p-1}^{\prime}\right\|+\alpha_{n, p-2} \alpha_{n, p-1} \alpha_{n, p} L^{2} \gamma^{2}\left\|e_{n, p}^{\prime}\right\| \\
& +\left(\alpha_{n, p-2} L+\alpha_{n, p-2} \alpha_{n, p-1} L^{2} \gamma+\alpha_{n, p-2} \alpha_{n, p-1} \alpha_{n, p} L^{3} \gamma^{2}\right) b_{n} \\
& +\left\|e_{n, p-2}^{\prime \prime}\right\|+\alpha_{n, p-2} L \gamma\left\|e_{n, p-1}^{\prime \prime}\right\|+\alpha_{n, p-2} \alpha_{n, p-1} L^{2} \gamma^{2}\left\|e_{n, p}^{\prime \prime}\right\| \\
& +\left\|r_{n, p-2}\right\|+\alpha_{n, p-2} L \gamma\left\|r_{n, p-1}\right\|+\alpha_{n, p-2} \alpha_{n, p-1} L^{2} \gamma^{2}\left\|r_{n, p}\right\| \\
& +\left(\beta_{n, p-2}+\alpha_{n, p-2} \beta_{n, p-1} L \gamma+\alpha_{n, p-2} \alpha_{n, p-1} \beta_{n, p} L^{2} \gamma^{2}\right) \Gamma .
\end{aligned}
$$

Continuing this procedure in (4.10)-(4.12), we gain

$$
\begin{aligned}
\left\|\gamma_{n, 1}-x^{*}\right\| \leq & \left(1-\alpha_{n, 2}-\beta_{n, 2}+\alpha_{n, 2}\left(1-\alpha_{n, 3}-\beta_{n, 3}\right) L \gamma+\alpha_{n, 2} \alpha_{n, 3}\left(1-\alpha_{n, 4}-\beta_{n, 4}\right) L^{2} \gamma^{2}\right. \\
& \left.+\cdots+\prod_{i=2}^{p-1} \alpha_{n, i}\left(1-\alpha_{n, p}-\beta_{n, p}\right) L^{p-2} \gamma^{p-2}+\prod_{i=2}^{p} \alpha_{n, i} L^{p-1} \gamma^{p-1}\right)\left\|x_{n}-x^{*}\right\| \\
& +\left(\alpha_{n, 2} L+\alpha_{n, 2} \alpha_{n, 3} L^{2} \gamma+\alpha_{n, 2} \alpha_{n, 3} \alpha_{n, 4} L^{3} \gamma^{2}+\cdots+\prod_{i=2}^{p} \alpha_{n, i} L^{p-1} \gamma^{p-2}\right) b_{n} \\
& +\alpha_{n, 2}\left\|e_{n, 2}^{\prime}\right\|+\alpha_{n, 2} \alpha_{n, 3} L \gamma\left\|e_{n, 3}^{\prime}\right\|+\cdots+\prod_{i=2}^{p} \alpha_{n, i} L^{p-2} \gamma^{p-2}\left\|e_{n, p}^{\prime}\right\| \\
& +\left\|e_{n, 2}^{\prime \prime}\right\|+\alpha_{n, 2} L \gamma\left\|e_{n, 3}^{\prime \prime}\right\|+\alpha_{n, 2} \alpha_{n, 3} L^{2} \gamma^{2}\left\|e^{\prime \prime}{ }_{n, 4}\right\|+\cdots+\prod_{i=2}^{p-1} \alpha_{n, i} L^{p-2} \gamma^{p-2}\left\|e_{n, p}^{\prime \prime}\right\| \\
& +\left\|r_{n, 2}\right\|+\alpha_{n, 2} L \gamma\left\|r_{n, 3}\right\|+\alpha_{n, 2} \alpha_{n, 3} L^{2} \gamma^{2}\left\|r_{n, 4}\right\|+\cdots+\prod_{i=2}^{p-1} \alpha_{n, i} L^{p-2} \gamma^{p-2}\left\|r_{n, p}\right\| \\
& +\left(\beta_{n, 2}+\alpha_{n, 2} \beta_{n, 3} L \gamma+\alpha_{n, 2} \alpha_{n, 3} \beta_{n, 4} L^{2} \gamma^{2}+\cdots+\prod_{i=2}^{p-1} \alpha_{n, i} \beta_{n, p} L^{p-2} \gamma^{p-2}\right) \Gamma .
\end{aligned}
$$


It follows from (4.8) and (4.13) that

$$
\begin{aligned}
& \left\|x_{n+1}-x^{*}\right\| \leq\left(1-\alpha_{n, 1}-\beta_{n, 1}\right)\left\|x_{n}-x^{*}\right\|+\alpha_{n, 1} L \gamma\left\|y_{n, 1}-x^{*}\right\| \\
& +\alpha_{n, 1} L b_{n}+\alpha_{n, 1}\left\|e_{n, 1}^{\prime}\right\|+\left\|e_{n, 1}^{\prime \prime}\right\|+\left\|r_{n, 1}\right\|+\beta_{n, 1} \Gamma \\
& \leq\left(1-\alpha_{n, 1}-\beta_{n, 1}+\alpha_{n, 1}\left(1-\alpha_{n, 2}-\beta_{n, 2}\right) L \gamma+\alpha_{n, 1} \alpha_{n, 2}\left(1-\alpha_{n, 3}-\beta_{n, 3}\right) L^{2} \gamma^{2}\right. \\
& \left.+\cdots+\prod_{i=1}^{p-1} \alpha_{n, i}\left(1-\alpha_{n, p}-\beta_{n, p}\right) L^{p-1} \gamma^{p-1}+\prod_{i=1}^{p} \alpha_{n, i} L^{p} \gamma^{p}\right)\left\|x_{n}-x^{*}\right\| \\
& +\left(\alpha_{n, 1} L+\alpha_{n, 1} \alpha_{n, 2} L^{2} \gamma+\alpha_{n, 1} \alpha_{n, 2} \alpha_{n, 3} L^{3} \gamma^{2}+\cdots+\prod_{i=1}^{p} \alpha_{n, i} L^{p} \gamma^{p-1}\right) b_{n} \\
& +\alpha_{n, 1}\left\|e_{n, 1}^{\prime}\right\|+\alpha_{n, 1} \alpha_{n, 2} L \gamma\left\|e_{n, 2}^{\prime}\right\|+\cdots+\prod_{i=1}^{p} \alpha_{n, i} L^{p-1} \gamma^{p-1}\left\|e_{n, p}^{\prime}\right\| \\
& +\left\|e_{n, 1}^{\prime \prime}\right\|+\alpha_{n, 1} L \gamma\left\|e_{n, 2}^{\prime \prime}\right\|+\alpha_{n, 1} \alpha_{n, 2} L^{2} \gamma^{2}\left\|e^{\prime \prime}{ }_{n, 3}\right\|+\cdots+\prod_{i=1}^{p-1} \alpha_{n, i} L^{p-1} \gamma^{p-1}\left\|e^{\prime \prime}{ }_{n, p}\right\| \\
& +\left\|r_{n, 1}\right\|+\alpha_{n, 1} L \gamma\left\|r_{n, 2}\right\|+\alpha_{n, 1} \alpha_{n, 2} L^{2} \gamma^{2}\left\|r_{n, 3}\right\|+\cdots+\prod_{i=1}^{p-1} \alpha_{n, i} L^{p-1} \gamma^{p-1}|| r_{n, p} \| \\
& +\left(\beta_{n, 1}+\alpha_{n, 1} \beta_{n, 2} L \gamma+\alpha_{n, 1} \alpha_{n, 2} \beta_{n, 3} L^{2} \gamma^{2}+\cdots+\prod_{i=1}^{p-1} \alpha_{n, i} \beta_{n, p} L^{p-1} \gamma^{p-1}\right) \Gamma \\
& \leq\left[1-(1-L \gamma) \prod_{i=1}^{p} \alpha_{n, i} L^{p-1} \gamma^{p-1}\right]\left\|x_{n}-x^{*}\right\|+\sum_{i=1}^{p} \prod_{j=1}^{i} \alpha_{n, j} L^{i} \gamma^{i-1} b_{n} \\
& +\sum_{i=1}^{p} \prod_{j=1}^{i} \alpha_{n, j} L^{i-1} \gamma^{i-1}\left\|e_{n, i}^{\prime}\right\|+\left\|e_{n, 1}^{\prime \prime}\right\|+\sum_{i=2}^{p} \prod_{j=1}^{i-1} \alpha_{n, j} L^{i-1} \gamma^{i-1}\left\|e_{n, i}^{\prime \prime}\right\|+\left\|r_{n, 1}\right\| \\
& +\sum_{i=2}^{p} \prod_{j=1}^{i-1} \alpha_{n, j} L^{i-1} \gamma^{i-1}\left\|r_{n, i}\right\|+\left(\beta_{n, 1}+\sum_{i=2}^{p} \prod_{j=1}^{i-1} \alpha_{n, j} \beta_{n, i} L^{i-1} \gamma^{i-1}\right) \Gamma \\
& =\left[1-(1-L \gamma) \prod_{i=1}^{p} \alpha_{n, i} L^{p-1} \gamma^{p-1}\right]\left\|x_{n}-x^{*}\right\| \\
& +(1-L \gamma) \prod_{i=1}^{p} \alpha_{n, i} L^{p-1} \gamma^{p-1} \frac{\sum_{i=1}^{p} \prod_{j=1}^{i} \alpha_{n, j} L^{i} \gamma^{i-1} b_{n}+\sum_{i=1}^{p} \prod_{j=1}^{i} \alpha_{n, j} L^{i-1} \gamma^{i-1}\left\|e_{n, i}^{\prime}\right\|}{\alpha(1-L \gamma) L^{p-1} \gamma^{p-1}} \\
& +\sum_{i=2}^{p} \prod_{j=1}^{i-1} \alpha_{n, j} L^{i-1} \gamma^{i-1}\left\|e^{\prime \prime}{ }_{n, i}\right\|+\sum_{i=2}^{p} \prod_{j=1}^{i-1} \alpha_{n, j} L^{i-1} \gamma^{i-1}\left\|r_{n, i}\right\| \\
& +\left\|e_{n, 1}^{\prime \prime}\right\|+\left\|r_{n, 1}\right\|+\left(\beta_{n, 1}+\sum_{i=2}^{p} \prod_{j=1}^{i-1} \alpha_{n, j} \beta_{n, i} L^{i-1} \gamma^{i-1}\right) \Gamma .
\end{aligned}
$$

Since $L \gamma<1$ and $\lim _{n \rightarrow \infty} b_{n}=0$, in view of (4.3), it is clear that all the conditions of Lemma 4.7 are satisfied and so Lemma 4.7 and (4.14) guarantee that $x_{n} \rightarrow x^{*}$, as $n \rightarrow$ $\infty$. Thus the sequence $\left\{x_{n}\right\}_{n=0}^{\infty}$ generated by Algorithm 4.1 converges strongly to the only element of $\operatorname{Fix}(S) \cap \operatorname{EGNRNVID}\left(K_{r}, T, f, g\right)$. This completes the proof.

As in the proof of Theorem 3.5, one can prove the convergence of iterative sequences generated by Algorithms 4.2-4.4 and we omit their proofs.

Theorem 4.9. Assume that T, $f$ and $\rho$ are the same as in Theorem 3.4 such that the conditions (a), (b) and (3.18) in Theorem 3.4 hold. Let $S: K_{r} \rightarrow K_{r}$ be a nearly uniformly L-Lipschitzian mapping with the sequence $\left\{b_{n}\right\}_{n=0}^{\infty}$ such that $\operatorname{Fix}(S) \cap$ $\operatorname{GNRNVID}\left(K_{r}, T, f\right) \neq \varnothing$, where GNRNVID $\left(K_{r}, T, f\right)$ is the set of the solutions of the problem (3.3). Further, let $L \theta<1$, where $\theta=\frac{r}{r-r^{\prime}} \sqrt{\varpi^{2}-2 \rho \kappa+\rho^{2} \sigma^{2}}$. If there exists a constant $\alpha>0$ such that $\prod_{i=1}^{p} \alpha_{n, i}>\alpha$ for each $n \geq 0$, then the iterative sequence $\left\{x_{n}\right\}_{n=0}^{\infty}$ generated by Algorithm 4.2 converges strongly to the only element of $\operatorname{Fix}(S) \cap$ GNRNVID $\left(K_{r}, T, f\right)$. 
Theorem 4.10. Suppose that $T, g$ and $\rho$ are the same as in Theorem 3.5 such that the conditions (a), (b) and (3.19) in Theorem 3.5 hold. Let $S: K_{r} \rightarrow K_{r}$ be a nearly uniformly L-Lipschitzian mapping with the sequence $\left\{b_{n}\right\}_{n=0}^{\infty}$ such that $\operatorname{Fix}(S) \cap$ GNRNVID $\left(K_{r}, T, f\right) \neq \varnothing$, where GNRNVID $\left(K_{r}, T, g\right)$ is the set of the solutions of the problem (3.4). Further, let $L \tilde{\theta}<1$, where

$$
\tilde{\theta}=\sqrt{1-2 \tau+\iota^{2}}+\frac{r}{r-r^{\prime}} \sqrt{\iota^{2}-2 \rho \kappa+\rho^{2} \sigma^{2}} .
$$

If there exists a constant $\alpha>0$ such that $\prod_{i=1}^{p} \alpha_{n, i}>\alpha$ for each $n \geq 0$, then the iterative sequence $\left\{x_{n}\right\}_{n=0}^{\infty}$ generated by Algorithm 4.3 converges strongly to the only element of $\operatorname{Fix}(S) \cap$ GNRNVID $\left(K_{r}, T, g\right)$.

Theorem 4.11. Let $T$ and $\rho$ be the same as in Theorem 3.6 such that the condition (3.20) in Theorem 3.6 holds. Assume that $S: K_{r} \rightarrow K_{r}$ is a nearly uniformly L-Lipschitzian mapping with the sequence $\left\{b_{n}\right\}_{n=0}^{\infty}$ such that $\operatorname{Fix}(S) \cap \operatorname{NRNVI}\left(K_{r}, T\right) \neq \varnothing$, where NRNVI $\left(K_{r}, T\right)$ is the set of the solutions of the problem (3.5). Moreover, let $L \eta<1$, where $\eta=\frac{r}{r-r^{\prime}} \sqrt{1-2 \rho \kappa+\rho^{2} \sigma^{2}}$. Then the iterative sequence $\left\{x_{n}\right\}_{n=0}^{\infty}$ generated by Algorithm 4.4 converges strongly to the only element of $\operatorname{Fix}(S) \cap$ NRNVI $\left(K_{r}, T\right)$.

\section{Extended general nonconvex Wiener-Hopf equations}

In this section, we introduce a new class of extended general nonconvex Wiener-Hopf equations and some new special cases from it, and by using the projection method, we establish that the aforesaid class is equivalent with the class of extended general nonlinear regularized nonconvex variational inequalities (3.1).

Let $T, f, g$ and $\rho$ be the same as in the problem (3.1) and suppose that the inverse of the operator $g$ exists. Associated with the problem (3.1), the problem of finding $z \in \mathcal{H}$ such that

$$
T g^{-1} P_{K_{r}} z+\rho^{-1} Q_{K_{r}} z=0,
$$

Where $Q_{K_{r}}=I-f g^{-1} P_{K_{r}}$ with $I$ the identity operator and $P_{K_{r}}$ the projection operator is considered.

The problem (5.1) is called the extended general nonconvex Wiener-Hopf equation (EGNWHE) associated with the problem of extended general nonlinear regularized nonconvex variational inequality (3.1). Next, we denote by $\operatorname{EGNWHE}\left(K_{r}, T, f, g\right)$ the set of the solutions of the extended general nonconvex Wiener-Hopf equation (5.1).

Now, we state some special cases of the problem (5.1) as follows:

(1) If $g \equiv I$, then the problem (5.1) is equivalent to the following problem: Find $z \in \mathcal{H}$ such that

$$
T P_{K_{r}} z+\rho^{-1} Q_{K_{r}} z=0,
$$

where $Q_{K_{r}}=I-f P_{K_{r}}$ and is called the general nonconvex Wiener-Hopf equation (GNWHE) associated with the problem of general nonlinear regularized nonconvex variational inequality (3.3). We denote by $\operatorname{GNWHE}\left(K_{r}, T, f\right)$ the set of the solutions of the general nonconvex Wiener-Hopf equation (5.2). 
(2) If $f=g$, then the problem (5.1) changes into the following problem: Find $z \in \mathcal{H}$ such that

$$
T g^{-1} P_{K_{r}} z+\rho^{-1} Q_{K_{r}} z=0,
$$

where $Q_{K_{r}}=I-P_{K_{r}}$ and is called also the general nonconvex Wiener-Hopf equation (GNWHE) associated with the problem of general nonlinear regularized nonconvex variational inequality (3.4). We denote by GNWHE $\left(K_{r}, T, g\right)$ the set of the solutions of the general nonconvex Wiener-Hopf equation (5.2).

(3) If $f=g \equiv I$, then the problem (5.1) collapses to the following problem: Find $z \in \mathcal{H}$ such that

$$
T P_{K_{r}} z+\rho^{-1} Q_{K_{r}} z=0,
$$

where $Q_{K_{r}}$ is the same as in Eq. (5.3). The equation of the type (5.4) is called the nonconvex Wiener-Hopf equation (NWHE) associated with the problem of nonlinear regularized nonconvex variational inequality (3.4).

We denote by NWHE $\left(K_{r}, T\right)$ the set of the solutions of the nonconvex WienerHopf equation (5.2).

(4) If $r=\infty$, that is $K_{r}=K$, then the problem (5.1) reduces to the following problem: Find $z \in \mathcal{H}$ such that

$$
\mathrm{Tg}^{-1} P_{K} z+\rho^{-1} Q_{K} z=0,
$$

Where $Q_{K}=I-f_{g}^{-1} P_{K}$. The equations of the type (5.5) were introduced and studied by Noor [39].

(5) If $r=\infty$, then the problem (5.2) is equivalent to the following problem: Find $z \in \mathcal{H}$ such that

$$
T P_{K} z+\rho^{-1} Q_{K} z=0,
$$

where $Q_{K}=I-P_{K}$. The problem (5.6) is introduced and studied by Noor [35].

(6) If $r=\infty$, then the problem (5.3) changes into the following problem: Find $z \in \mathcal{H}$ such that

$$
\mathrm{Tg}^{-1} P_{K} z+\rho^{-1} Q_{K} z=0,
$$

where $Q_{K}=I-P_{K}$. The equations of the type (5.7) were introduced and studied by Noor [40].

(7) If $r=\infty$, then the problem (5.4) reduces to the following problem: Find $z \in \mathcal{H}$ such that

$$
T P_{K} z+\rho^{-1} Q_{K} z=0,
$$

where $Q_{K}$ is the same as in (5.7). The equation (5.8) is the original Wiener-Hopf equation mainly due to Shi [50]. 
Remark 5.1. It has been shown that the Wiener-Hopf equations have played an important and significant role in developing several numerical techniques for solving variational inequalities and related optimizations problems (see, for example, $[30,31,35,39,50]$ and references therein).

The following lemma shows that the extended general nonlinear regularized nonconvex variational inequality (3.1) and the extended general nonconvex Wiener-Hopf equation (5.1) are equivalent.

Lemma 5.2. Let T, $f, g$ and $\rho$ be the same as in the problem (3.1) and suppose that the inverse of the operator $g$ exists. Then $u \in \mathcal{H}$ with $g(u) \in K_{r}$ is a solution of the problem (3.1) if and only if the extended general nonconvex Wiener-Hopf equation (5.1) has a solution $z \in \mathcal{H}$ satisfying the following:

$$
g(u)=P_{K_{r}} z, \quad z=f(u)-\rho T(u) .
$$

Proof. Let $u \in \mathcal{H}$ with $g(u) \in K_{r}$ be a solution of the problem (3.1). Then, from Lemma 3.2, it follows that

$$
g(u)=P_{K_{r}}(f(u)-\rho T(u)) .
$$

Taking $z=f(u)-\rho T(u)$ in (5.9), we have $g(u)=P_{K_{r}} z$, which leads to

$$
u=g^{-1} P_{K_{r}} z \text {. }
$$

Applying (5.10) and this fact that $z=f(u)-\rho T(u)$, we have

$$
z=f g^{-1} P_{K_{r}} z-\rho T g^{-1} P_{K_{r}} z .
$$

Evidently, the above equality is equivalent to the following:

$$
T g^{-1} P_{K_{r}} z+\rho^{-1} Q_{K_{r}} z=0,
$$

where $Q_{K_{r}}$ is the same as in (5.1). Now, (5.11) guarantees that $z \in \mathcal{H}$ is a solution of the extended general nonconvex Wiener-Hopf equation (5.1).

Conversely, if $z \in \mathcal{H}$ is a solution of the problem (5.1) satisfying the following:

$$
g(u)=P_{K_{r}} z, \quad z=f(u)-\rho T(u),
$$

then it follows from Lemma 3.2 that $u \in \mathcal{H}$ with $g(u) \in K_{r}$ is a solution of the problem (3.1). This completes the proof.

In similar way to the proof of Lemma 5.2, one can prove the following statements.

Lemma 5.3. Let $T, f$ and $\rho$ be the same as in the problem (3.3). Then $u \in K_{r}$ is a solution of the problem (3.3) if and only if the general nonconvex Wiener-Hopf equation (5.2) has a solution $z \in \mathcal{H}$ satisfying

$$
u=P_{K_{r}} z, \quad z=f(u)-\rho T(u) .
$$

Lemma 5.4. Suppose that $T, g$ and $\rho$ are the same as in the problem (3.4) and let the inverse of the operator $g$ exists. Then $u \in \mathcal{H}$ with $g(u) \in K_{r}$ is a solution of the problem (3.4) if and only if the general nonconvex Wiener-Hopf equation (5.3) has a solution $z \in \mathcal{H}$ satisfying the following:

$$
g(u)=P_{K_{r}} z, \quad z=g(u)-\rho T(u) .
$$


Lemma 5.5. Assume that $T$ and $\rho$ are the same as in the problem (3.5). Then $u \in K_{r}$ is a solution of the problem (3.5) if and only if the nonconvex Wiener-Hopf equation (5.4) has a solution $z \in \mathcal{H}$ satisfying the following:

$$
u=P_{K_{r}} z, \quad z=u-\rho T(u) .
$$

\section{Some new perturbed $p$-step projection iterative methods}

In this section, by using the problems (5.1)-(5.4) and four Lemmas 5.2-5.5, we get some fixed point formulations for constructing a number of the new perturbed $p$-step projection iterative algorithms with mixed errors for solving the problems (3.1) and (3.3)-(3.5).

(I) By using (5.1) and Lemma 5.2, we have

$$
\begin{aligned}
\mathrm{Tg}^{-1} P_{K_{r}} z+\rho^{-1} Q_{K_{r}} z=0 & \Leftrightarrow \rho T g^{-1} P_{K_{r}} z+Q_{K_{r}} z=0 \\
& \Leftrightarrow \rho T g^{-1} P_{K_{r}} z+z-f g^{-1} P_{K_{r}} z=0 \\
& \Leftrightarrow z=f g^{-1} P_{K_{r}} z-\rho T g^{-1} P_{K_{r}} z \\
& \Leftrightarrow z=f(u)-\rho T(u) .
\end{aligned}
$$

This fixed point formulation enables us to define the following $p$-step projection iterative algorithm with mixed errors for solving the problem (3.1).

Algorithm 6.1. Let $T, f, g$ and $\rho$ be the same as in the problem (3.1) such that $g$ be an onto operator. For arbitrary chosen initial point $z_{0} \in \mathcal{H}$, compute the iterative sequence $\left\{z_{n}\right\}_{n=0}^{\infty}$ by

$$
\left\{\begin{array}{l}
g\left(u_{n}\right)=S^{n} P_{K_{r}} z_{n} \\
z_{n+1}=\left(1-\alpha_{n, 1}-\beta_{n, 1}\right) z_{n}+\alpha_{n, 1}\left(f\left(v_{n, 1}\right)-\rho T\left(v_{n, 1}\right)+e_{n, 1}\right)+\beta_{n, 1} l_{n, 1}+r_{n, 1} \\
v_{n, i}=\left(1-\alpha_{n, i+1}-\beta_{n, i+1}\right) z_{n}+\alpha_{n, i+1}\left(f\left(v_{n, i+1}\right)-\rho T\left(v_{n, i+1}\right)+e_{n, i+1}\right)+\beta_{n, i+1} l_{n, i+1}+r_{n, i+1} \\
\cdots \\
v_{n, p-1}=\left(1-\alpha_{n, p}-\beta_{n, p}\right) z_{n}+\alpha_{n, p}\left(f\left(u_{n}\right)-\rho T\left(u_{n}\right)+e_{n, p}\right)+\beta_{n, p} l_{n, p}+r_{n, p} \\
i=1,2, \ldots, p-2
\end{array}\right.
$$

where $S,\left\{\alpha_{n, i}\right\}_{n=0}^{\infty},\left\{\beta_{n, i}\right\}_{n=0}^{\infty},\left\{e_{n, i}\right\}_{n=0}^{\infty},\left\{l_{n, i}\right\}_{n=0}^{\infty},\left\{r_{n, i}\right\}_{n=0}^{\infty}(i=1,2, \ldots, p)$, are the same as in Algorithm 4.1.

(II) From (5.1) and Lemma 5.2, it follows that

$$
\begin{aligned}
\mathrm{Tg}^{-1} P_{K_{r}} z+\rho^{-1} Q_{K_{r}} z=0 & \Leftrightarrow Q_{K_{r}} z=Q_{K_{r}} z-T g^{-1} P_{K_{r}} z-\rho^{-1} Q_{K_{r}} z \\
& \Leftrightarrow Q_{K_{r}} z=-T g^{-1} P_{K_{r}} z+\left(1-\rho^{-1}\right) Q_{K_{r}} z \\
& \Leftrightarrow z=f g^{-1} P_{K_{r}} z-T g^{-1} P_{K_{r}} z+\left(1-\rho^{-1}\right) Q_{K_{r}} z \\
& \Leftrightarrow z=f(u)-T(u)+\left(1-\rho^{-1}\right) Q_{K_{r}} z .
\end{aligned}
$$

By using this fixed point formulation, we can construct the following $p$-step projection iterative algorithm with mixed errors for solving the problem (3.1).

Algorithm 6.2. Assume that $T, f, g$ and $\rho$ are the same as in Algorithm 6.1. For arbitrary chosen initial point $z_{0} \in \mathcal{H}$, compute the iterative sequence $\left\{z_{n}\right\}_{n=0}^{\infty}$ as follows:

$$
\left\{\begin{array}{l}
g\left(u_{n}\right)=S^{n} P_{K_{r}} z_{n \prime} \\
z_{n+1}=\left(1-\alpha_{n, 1}-\beta_{n, 1}\right) z_{n}+\alpha_{n, 1}\left(\Psi\left(v_{n, 1}, z_{n}\right)+e_{n, 1}\right)+\beta_{n, 1} l_{n, 1}+r_{n, 1} \\
v_{n, i}=\left(1-\alpha_{n, i+1}-\beta_{n, i+1}\right) z_{n}+\alpha_{n, i+1}\left(\Psi\left(v_{n, i+1}, z_{n}\right)+e_{n, i+1}\right)+\beta_{n, i+1} l_{n, i+1}+r_{n, i+1} \\
\cdots \\
v_{n, p-1}=\left(1-\alpha_{n, p}-\beta_{n, p}\right) z_{n}+\alpha_{n, p}\left(\Psi\left(u_{n,} z_{n}\right)+e_{n, p}\right)+\beta_{n, p} l_{n, p}+r_{n, p} \\
i=1,2, \ldots, p-2
\end{array}\right.
$$


where

$$
\left\{\begin{array}{l}
\Psi\left(v_{n, i}, z_{n}\right)=f\left(v_{n, i}\right)-T\left(v_{n, i}\right)+\left(1-\rho^{-1}\right) Q_{K_{r}} z_{n} \\
\Psi\left(u_{n}, z_{n}\right)=f\left(u_{n}\right)-T\left(u_{n}\right)+\left(1-\rho^{-1}\right) Q_{K_{r}} z_{n} \\
i=1,2, \ldots, p-2
\end{array}\right.
$$

and $S,\left\{\alpha_{n, i}\right\}_{n=0}^{\infty},\left\{\beta_{n, i}\right\}_{n=0}^{\infty},\left\{e_{n, i}\right\}_{n=0}^{\infty},\left\{l_{n, i}\right\}_{n=0}^{\infty},\left\{r_{n, i}\right\}_{n=0}^{\infty}(i=1,2, \ldots, p)$ are the same as in Algorithm 4.1.

(III) Like in the proof (I), by using (5.2) and Lemma 5.3, we have

$$
T P_{K_{r}} z+\rho^{-1} Q_{K_{r}} z=0 \quad \Leftrightarrow \quad z=f(u)-\rho T(u) .
$$

This fixed point formulation allows us to construct the following $p$-step projection iterative algorithm with mixed errors for solving the problem (3.3).

Algorithm 6.3. Let $T, f$ and $\rho$ be the same as in the problem (3.3). For arbitrary chosen initial point $z_{0} \in \mathcal{H}$, compute the iterative sequence $\left\{z_{n}\right\}_{n=0}^{\infty}$ by

$$
\left\{\begin{array}{l}
u_{n}=S^{n} P_{K_{r}} z_{n,} \\
z_{n+1}=\left(1-\alpha_{n, 1}-\beta_{n, 1}\right) z_{n}+\alpha_{n, 1}\left(f\left(v_{n, 1}\right)-\rho T\left(v_{n, 1}\right)+e_{n, 1}\right)+\beta_{n, 1} l_{n, 1}+r_{n, 1} \\
v_{n, i}=\left(1-\alpha_{n, i+1}-\beta_{n, i+1}\right) z_{n}+\alpha_{n, i+1}\left(f\left(v_{n, i+1}\right)-\rho T\left(v_{n, i+1}\right)+e_{n, i+1}\right)+\beta_{n, i+1} l_{n, i+1}+r_{n, i+1} \\
\cdots \\
v_{n, p-1}=\left(1-\alpha_{n, p}-\beta_{n, p}\right) z_{n}+\alpha_{n, p}\left(f\left(u_{n}\right)-\rho T\left(u_{n}\right)+e_{n, p}\right)+\beta_{n, p} l_{n, p}+r_{n, p} \\
i=1,2, \ldots, p-2,
\end{array}\right.
$$

where $S,\left\{\alpha_{n, i}\right\}_{n=0}^{\infty},\left\{\beta_{n, i}\right\}_{n=0}^{\infty},\left\{e_{n, i}\right\}_{n=0}^{\infty},\left\{l_{n, i}\right\}_{n=0}^{\infty},\left\{r_{n, i}\right\}_{n=0}^{\infty}(i=1,2, \ldots, p)$ are the same as in Algorithm 4.1.

(IV) In similar way, from (5.3) and Lemma 5.4, it follows that

$$
T g^{-1} P_{K_{r}} z+\rho^{-1} Q_{K_{r}} z=0 \quad \Leftrightarrow \quad z=g(u)-\rho T(u) .
$$

By using the above fixed point formulation, we can define the following $p$-step projection iterative algorithm with mixed errors for solving the problem (3.4).

Algorithm 6.4. Let $T, g$ and $\rho$ be the same as in the problem (3.4) such that $g$ be an onto operator. For arbitrary chosen initial point $z_{0} \in \mathcal{H}$, compute the iterative sequence $\left\{z_{n}\right\}_{n=0}^{\infty}$ by

$$
\left\{\begin{array}{l}
g\left(u_{n}\right)=S^{n} P_{K_{r}} z_{n,} \\
z_{n+1}=\left(1-\alpha_{n, 1}-\beta_{n, 1}\right) z_{n}+\alpha_{n, 1}\left(g\left(v_{n, 1}\right)-\rho T\left(v_{n, 1}\right)+e_{n, 1}\right)+\beta_{n, 1} l_{n, 1}+r_{n, 1}, \\
v_{n, i}=\left(1-\alpha_{n, i+1}-\beta_{n, i+1}\right) z_{n}+\alpha_{n, i+1}\left(g\left(v_{n, i+1}\right)-\rho T\left(v_{n, i+1}\right)+e_{n, i+1}\right)+\beta_{n, i+1} l_{n, i+1}+r_{n, i+1}, \\
\cdots \\
v_{n, p-1}=\left(1-\alpha_{n, p}-\beta_{n, p}\right) z_{n}+\alpha_{n, p}\left(g\left(u_{n}\right)-\rho T\left(u_{n}\right)+e_{n, p}\right)+\beta_{n, p} l_{n, p}+r_{n, p} \\
i=1,2, \ldots, p-2,
\end{array}\right.
$$

where $S,\left\{\alpha_{n, i}\right\}_{n=0}^{\infty},\left\{\beta_{n, i}\right\}_{n=0}^{\infty},\left\{e_{n, i}\right\}_{n=0}^{\infty},\left\{l_{n, i}\right\}_{n=0}^{\infty},\left\{l_{n, i}\right\}_{n=0}^{\infty}$ are the same as in Algorithm 4.1.

(V) Similarly, by using (5.4) and Lemma 5.5, we have

$$
T P_{K_{r}} z+\rho^{-1} Q_{K_{r}} z=0 \quad \Leftrightarrow \quad z=f(u)-\rho T(u) .
$$


This fixed point formulation enables us to construct the following $p$-step projection iterative algorithm with mixed errors for solving the problem (3.5).

Algorithm 6.5. Let $T$ and $\rho$ be the same as in the problem (3.5). For arbitrary chosen initial point $z_{0} \in \mathcal{H}$, compute the iterative sequence $\left\{z_{n}\right\}_{n=0}^{\infty}$ by the iterative scheme

$$
\left\{\begin{array}{l}
u_{n}=S^{n} P_{K_{r}} z_{n} \\
z_{n+1}=\left(1-\alpha_{n, 1}-\beta_{n, 1}\right) z_{n}+\alpha_{n, 1}\left(v_{n, 1}-\rho T\left(v_{n, 1}\right)+e_{n, 1}\right)+\beta_{n, 1} l_{n, 1}+r_{n, 1} \\
v_{n, i}=\left(1-\alpha_{n, i+1}-\beta_{n, i+1}\right) z_{n}+\alpha_{n, i+1}\left(v_{n, i+1}-\rho T\left(v_{n, i+1}\right)+e_{n, i+1}\right)+\beta_{n, i+1} l_{n, i+1}+r_{n, i+1} \\
\cdots \\
v_{n, p-1}=\left(1-\alpha_{n, p}-\beta_{n, p}\right) z_{n}+\alpha_{n, p}\left(u_{n}-\rho T\left(u_{n}\right)+e_{n, p}\right)+\beta_{n, p} l_{n, p}+r_{n, p}, \\
i=1,2, \ldots, p-2
\end{array}\right.
$$

where $S,\left\{\alpha_{n, i}\right\}_{n=0}^{\infty},\left\{\beta_{n, i}\right\}_{n=0}^{\infty},\left\{e_{n, i}\right\}_{n=0}^{\infty},\left\{l_{n, i}\right\}_{n=0}^{\infty},\left\{r_{n, i}\right\}_{n=0}^{\infty}(i=1,2, \ldots, p)$ are the same as in Algorithm 4.1.

Remark 6.6. In similar to Remark 4.5, for a suitable and appropriate choice of the sequences $\left\{e_{n, i}\right\}_{n=0}^{\infty},\left\{l_{n, i}\right\}_{n=0}^{\infty}$ and $\left\{r_{n, i}\right\}_{n=0}^{\infty}(i=1,2, \ldots, p)$, Algorithms 6.1-6.5 reduce to algorithms with mean errors and without errors.

Remark 6.7. Algorithm 3.1 in [42] is a special case of Algorithms 6.1 and 6.4. Algorithm 3.2 in [42] is a special case of Algorithm 6.2. Also, Algorithms 3.1-3.3 in [44] and Algorithms 2.1-2.3 in [47] are special cases of Algorithms 6.1, 6.2 and 6.4.

Now, we discuss the convergence analysis of iterative sequences generated by perturbed projection iterative Algorithms 6.1-6.5.

Theorem 6.8. Let T, $f, g$ and $\rho$ be the same as in the problem (3.1) and suppose that all the conditions of Theorem 3.3 hold. Assume that $S: K_{r} \rightarrow K_{r}$ is a nearly uniformly L-Lipschitzian mapping with the sequence $\left\{b_{n}\right\}_{n=0}^{\infty}$ such that, for any $u \in$ EGNRNVID $\left(K_{r}, T, f, g\right), g(u) \in \operatorname{Fix}(S)$. Further, assume that $L \gamma<1$, where $\gamma$ is the same as in (3.17). If there exists a constant $\alpha>0$ such that $\prod_{i=1}^{p} \alpha_{n, i}>\alpha$ for each $n \geq 0$, then the iterative sequence $\left\{z_{n}\right\}_{n=0}^{\infty}$ generated by Algorithm 6.1 converges strongly to the only element of $\operatorname{EGNWHE}\left(K_{r}, T, f, g\right)$.

Proof. Theorem 3.3 guarantees the existence a unique solution $u^{*} \in \mathcal{H}$ with $g\left(u^{*}\right) \in$ $K_{r}$ for the problem (3.1). Hence, in view of Lemma 5.2, there exists a unique point $z \in \mathcal{H}$ satisfying the following:

$$
g\left(u^{*}\right)=P_{K_{r}} z, \quad z=f\left(u^{*}\right)-\rho T\left(u^{*}\right) .
$$

Since $g\left(u^{*}\right) \in \operatorname{Fix}(S)$, it follows from (6.2) that, for each $n \geq 0$,

$$
g\left(u^{*}\right)=S^{n} P_{K_{r}} z, \quad z=f\left(u^{*}\right)-\rho T\left(u^{*}\right) .
$$

Let $\Gamma=\sup _{n \geq 0}\left\{|| l_{n, i}-z||,|| z-u^{*}||: i=1,2, \ldots, p\right\}$. By using (6.1), (6.2) and the assumptions, we have 


$$
\begin{aligned}
\left\|z_{n+1}-z\right\| \leq & \left(1-\alpha_{n, 1}-\beta_{n, 1}\right)\left\|z_{n}-z\right\|+\alpha_{n, 1}|| f\left(v_{n, 1}\right)-f\left(u^{*}\right)-\rho\left(T\left(v_{n, 1}\right)-T\left(u^{*}\right)\right) \| \\
& +\beta_{n, 1}|| l_{n, 1}-z\left\|+\alpha_{n, 1}|| e_{n, 1}\right\|+\left\|r_{n, 1}\right\| \\
\leq & \left(1-\alpha_{n, 1}-\beta_{n, 1}\right)\left\|z_{n}-z\right\|+\alpha_{n, 1} \sqrt{\varpi^{2}-2 \rho \kappa+\rho^{2} \sigma^{2}}|| v_{n, 1}-u^{*} \| \\
& +\beta_{n, 1}|| l_{n, 1}-z\left\|+\alpha_{n, 1}\left(\left\|e_{n, 1}^{\prime}\right\|+\left\|e^{\prime \prime}{ }_{n, 1}\right\|\right)+\right\| r_{n, 1} \| \\
\leq & \left(1-\alpha_{n, 1}-\beta_{n, 1}\right)\left\|z_{n}-z\right\|+\alpha_{n, 1} \sqrt{\varpi^{2}-2 \rho \kappa+\rho^{2} \sigma^{2}}|| v_{n, 1}-z \| \\
& +\alpha_{n, 1} \sqrt{\varpi^{2}-2 \rho \kappa+\rho^{2} \sigma^{2}}|| z-u^{*}\left\|+\alpha_{n, 1}\right\| e_{n, 1}^{\prime}\|+\| e^{\prime \prime}{ }_{n, 1}\|+\| r_{n, 1} \|+\beta_{n, 1} \Gamma \\
\leq & \left(1-\alpha_{n, 1}-\beta_{n, 1}\right)|| z_{n}-z\left\|+\alpha_{n, 1} \sqrt{\varpi^{2}-2 \rho \kappa+\rho^{2} \sigma^{2}}|| v_{n, 1}-z\right\| \\
& +\left(\alpha_{n, 1} \sqrt{\varpi^{2}-2 \rho \kappa+\rho^{2} \sigma^{2}}+\beta_{n, 1}\right) \Gamma+\alpha_{n, 1}|| e_{n, 1}^{\prime}\|+\| e^{\prime \prime}{ }_{n, 1}\|+\| r_{n, 1} \| .
\end{aligned}
$$

In similar way to the proof (6.4), for each $i \in\{1,2, \ldots, p-2\}$, we can get

$$
\begin{aligned}
\left\|v_{n, i}-z\right\| \leq & \left(1-\alpha_{n, i+1}-\beta_{n, i+1}\right)\left\|z_{n}-z\right\| \\
& +\alpha_{n, i+1} \sqrt{\varpi^{2}-2 \rho \kappa+\rho^{2} \sigma^{2}}\left\|v_{n, i+1}-z\right\| \\
& +\left(\alpha_{n, i+1} \sqrt{\varpi^{2}-2 \rho \kappa+\rho^{2} \sigma^{2}}+\beta_{n, i+1}\right) \Gamma \\
& +\alpha_{n, i+1}\left\|e_{n, i+1}^{\prime}\right\|+\left\|e_{n, i+1}^{\prime \prime}\right\|+\left\|r_{n, i+1}\right\|
\end{aligned}
$$

and

$$
\begin{gathered}
\left\|v_{n, p-1}-z\right\| \leq\left(1-\alpha_{n, p}-\beta_{n, p}\right)\left\|z_{n}-z\right\|+\alpha_{n, p} \sqrt{\varpi^{2}-2 \rho \kappa+\rho^{2} \sigma^{2}}\left\|u_{n}-u^{*}\right\| \\
+\alpha_{n, p}\left\|e_{n, p}^{\prime}\right\|+\left\|e_{n, p}^{\prime \prime}\right\|+\left\|r_{n, p}\right\|+\beta_{n, p} \Gamma .
\end{gathered}
$$

Now, we make an estimation for $\left\|u_{n}-u^{*}\right\|$. Applying (6.1), (6.3) and Proposition 2.10 , we find that

$$
\begin{aligned}
\left\|u_{n}-u^{*}\right\| & \leq\left\|u_{n}-u^{*}-\left(g\left(u_{n}\right)-g\left(u^{*}\right)\right)\right\|+\left\|S^{n} P_{K_{r}} z_{n}-S^{n} P_{K_{r}} z\right\| \\
& \leq \sqrt{1-2 \tau+\iota^{2}}|| u_{n}-u^{*} \|+L\left(\frac{r}{r-r^{\prime}}|| z_{n}-z \|+b_{n}\right),
\end{aligned}
$$

which leads to

$$
\left\|u_{n}-u^{*}\right\| \leq \frac{r L}{\left(r-r^{\prime}\right)\left(1-\sqrt{1-2 \tau+\iota^{2}}\right)}\left\|z_{n}-z\right\|+\frac{L b_{n}}{1-\sqrt{1-2 \tau+\iota^{2}}} .
$$

By using (6.6) and (6.7), we conclude that

$$
\begin{aligned}
\left\|v_{n, p-1}-z\right\| \leq & \left(1-\alpha_{n, p}-\beta_{n, p}\right)\left\|z_{n}-z\right\| \\
& +\alpha_{n, p} L \frac{r \sqrt{\varpi^{2}-2 \rho \kappa+\rho^{2} \sigma^{2}}}{\left(r-r^{\prime}\right)\left(1-\sqrt{1-2 \tau+\iota^{2}}\right)}\left\|z_{n}-z\right\| \\
& +\alpha_{n, p} L \frac{\sqrt{\varpi^{2}-2 \rho \kappa+\rho^{2} \sigma^{2}}}{1-\sqrt{1-2 \tau+\iota^{2}}} b_{n} \\
& +\alpha_{n, p}\left\|e_{n, p}^{\prime}\right\|+\left\|e_{n, p}^{\prime \prime}\right\|+\left\|r_{n, p}\right\|+\beta_{n, p} \Gamma \\
\leq & \left(1-\alpha_{n, p}-\beta_{n, p}\right)\left\|z_{n}-z\right\|+\alpha_{n, p} L \vartheta\left\|z_{n}-z\right\| \\
& +\alpha_{n, p} \frac{\left(r-r^{\prime}\right) L \vartheta}{r} b_{n}+\alpha_{n, p}\left\|e_{n, p}^{\prime}\right\|+\left\|e^{\prime \prime}{ }_{n, p}\right\|+\left\|r_{n, p}\right\|+\beta_{n, p} \Gamma,
\end{aligned}
$$

where $\vartheta=\frac{r \sqrt{\varpi^{2}-2 \rho \kappa+\rho^{2} \sigma^{2}}}{\left(r-r^{\prime}\right)\left(1-\sqrt{1-2 \tau+\iota^{2}}\right)}$ In view of the condition (3.11), we have $\vartheta<1$. From $r^{\prime} \in$ $(0, r)$ and (6.8), we have 


$$
\begin{aligned}
\left\|v_{n, p-1}-z\right\| & \leq\left(1-\alpha_{n, p}-\beta_{n, p}\right)\left\|z_{n}-z\right\|+\alpha_{n, p} L \vartheta\left\|z_{n}-z\right\| \\
& +\alpha_{n, p} L \vartheta b_{n}+\alpha_{n, p}\left\|e_{n, p}^{\prime}\right\|+\left\|e_{n, p}^{\prime \prime}\right\|+\left\|r_{n, p}\right\|+\beta_{n, p} \Gamma .
\end{aligned}
$$

Since $\sqrt{1-2 \tau+\iota^{2}}+\frac{r}{r-r^{\prime}} \sqrt{\omega^{2}-2 \rho \kappa+\rho^{2} \sigma^{2}}<1$ and $r^{\prime} \in(0, r)$, deduce that

$$
\lambda=\sqrt{\varpi^{2}-2 \rho \kappa+\rho^{2} \sigma^{2}}<1 .
$$

By using (6.10), the inequality (6.5), for each $i=1,2, \ldots, p-2$, can be written as follows:

$$
\begin{aligned}
\left\|v_{n, i}-z\right\| \leq & \left(1-\alpha_{n, i+1}-\beta_{n, i+1}\right)\left\|z_{n}-z\right\|+\alpha_{n, i+1} \lambda\left\|v_{n, i+1}-z\right\| \\
& +\alpha_{n, i+1}\left\|e_{n, i+1}^{\prime}\right\|+\left\|e^{\prime \prime}{ }_{n, i+1}\right\|+\left\|r_{n, i+1}\right\|+\left(\alpha_{n, i+1} \lambda+\beta_{n, i+1}\right) \Gamma .
\end{aligned}
$$

Thus it follows from (6.9) and (6.11) that

$$
\begin{aligned}
\left\|v_{n, p-2}-z\right\| \leq & \left(1-\alpha_{n, p-1}-\beta_{n, p-1}\right)\left\|z_{n}-z\right\|+\alpha_{n, p-1} \lambda\left\{\left(1-\alpha_{n, p}-\beta_{n, p}\right)\left\|z_{n}-z\right\|\right. \\
& \left.+\alpha_{n, p} L \vartheta\left\|z_{n}-z\right\|+\alpha_{n, p} L \vartheta b_{n}+\alpha_{n, p}\left\|e_{n, p}\right\|+\left\|e_{n, p}^{\prime \prime}\right\|+\left\|r_{n, p}\right\|+\beta_{n, p} \Gamma\right\} \\
& +\alpha_{n, p-1}\left\|e_{n, p-1}^{\prime}\right\|+\left\|e_{n, p-1}^{\prime \prime}\right\|+\left\|r_{n, p-1}\right\|+\left(\alpha_{n, p-1} \lambda+\beta_{n, p-1}\right) \Gamma \\
& =\left(1-\alpha_{n, p-1}-\beta_{n, p-1}+\alpha_{n, p-1}\left(1-\alpha_{n, p}-\beta_{n, p}\right) \lambda+\alpha_{n, p-1} \alpha_{n, p} \lambda L \vartheta\right)\left\|z_{n}-z\right\| \\
& +\alpha_{n, p-1} \alpha_{n, p} \lambda L \vartheta b_{n}+\alpha_{n, p-1} \alpha_{n, p} \lambda\left\|e_{n, p}^{\prime}\right\|+\alpha_{n, p-1}\left\|e_{n, p-1}^{\prime}\right\| \\
& +\alpha_{n, p-1} \lambda\left\|e_{n, p}^{\prime \prime}\right\|+\left\|e_{n, p-1}^{\prime \prime}\right\|+\alpha_{n, p-1} \lambda\left\|\mid r_{n, p}\right\|+\left\|r_{n, p-1}\right\| \\
& +\left(\alpha_{n, p-1} \beta_{n, p} \lambda+\alpha_{n, p-1} \lambda+\beta_{n, p-1}\right) \Gamma .
\end{aligned}
$$

Similarly, by using (6.11) and (6.12), we obtain

$$
\begin{aligned}
\left\|v_{n, p-3}-z\right\| \leq & \left(1-\alpha_{n, p-2}-\beta_{n, p-2}+\alpha_{n, p-2}\left(1-\alpha_{n, p-1}-\beta_{n, p-1}\right) \lambda\right. \\
& \left.+\alpha_{n, p-2} \alpha_{n, p-1}\left(1-\alpha_{n, p}-\beta_{n, p}\right) \lambda^{2}+\alpha_{n, p-2} \alpha_{n, p-1} \alpha_{n, p} \lambda^{2} L \vartheta\right)\left\|z_{n}-z\right\| \\
& +\alpha_{n, p-2} \alpha_{n, p-1} \alpha_{n, p} \lambda^{2}\left\|e_{n, p}^{\prime}\right\|+\alpha_{n, p-2} \alpha_{n, p-1} \lambda\left\|e_{n, p-1}^{\prime}\right\|+\alpha_{n, p-2}\left\|e_{n, p-2}^{\prime}\right\| \\
& +\alpha_{n, p-2} \alpha_{n, p-1} \alpha_{n, p} \lambda^{2} L \vartheta b_{n}+\alpha_{n, p-2} \alpha_{n, p-1} \lambda^{2}\left\|e_{n, p}^{\prime \prime}\right\|+\alpha_{n, p-2} \lambda\left\|e_{n, p-1}^{\prime \prime}\right\|+\left\|e_{n, p-2}^{\prime \prime}\right\| \\
& +\alpha_{n, p-2} \alpha_{n, p-1} \lambda^{2}\left\|r_{n, p}\right\|+\alpha_{n, p-2} \lambda\left\|r_{n, p-1}\right\|+\left\|r_{n, p-2}\right\|+\left(\alpha_{n, p-2} \lambda+\alpha_{n, p-2} \alpha_{n, p-1} \lambda^{2}\right) \Gamma \\
& +\left(\beta_{n, p-2}+\alpha_{n, p-2} \beta_{n, p-1} \lambda+\alpha_{n, p-2} \alpha_{n, p-1} \beta_{n, p} \lambda^{2}\right) \Gamma .
\end{aligned}
$$

Continuing the same procedures, we get

$$
\begin{aligned}
\left\|v_{n, 1}-z\right\| \leq & \left(1-\alpha_{n, 2}-\beta_{n, 2}+\alpha_{n, 2}\left(1-\alpha_{n, 3}-\beta_{n, 3}\right) \lambda+\alpha_{n, 2} \alpha_{n, 3}\left(1-\alpha_{n, 4}-\beta_{n, 4}\right) \lambda^{2}\right. \\
& \left.+\cdots+\prod_{i=2}^{p-1} \alpha_{n, i}\left(1-\alpha_{n, p}-\beta_{n, p}\right) \lambda^{p-2}+\prod_{i=2}^{p} \alpha_{n, i} \lambda^{p-2} L \vartheta\right)\left\|z_{n}-z\right\| \\
& +\alpha_{n, 2}\left\|e_{n, 2}^{\prime}\right\|+\alpha_{n, 2} \alpha_{n, 3} \lambda\left\|e_{n, 3}^{\prime}\right\|+\alpha_{n, 2} \alpha_{n, 3} \alpha_{n, 4} \lambda^{2}\left\|e_{n, 4}^{\prime}\right\|+\cdots+\prod_{i=2}^{p} \alpha_{n, i} \lambda^{p-2}\left\|e_{n, p}^{\prime}\right\| \\
& +\prod_{i=2}^{p} \alpha_{n, i} \lambda^{p-2} L \vartheta b_{n}+\left\|e_{n, 2}^{\prime \prime}\right\|+\alpha_{n, 2} \lambda\left\|e_{n, 3}^{\prime \prime}\right\|+\alpha_{n, 2} \alpha_{n, 3} \lambda^{2}\left\|e_{n, 4}^{\prime \prime}\right\|+\cdots+\prod_{i=2}^{p-1} \alpha_{n, i} \lambda^{p-2}\left\|e_{n, p}^{\prime \prime}\right\| \\
& +\left\|r_{n, 2}\right\|+\alpha_{n, 2} \lambda\left\|r_{n, 3}\right\|+\alpha_{n, 2} \alpha_{n, 3} \lambda^{2}\left\|r_{n, 4}\right\|+\cdots+\prod_{i=2}^{p-1} \alpha_{n, i} \lambda^{p-2}\left\|r_{n, p}\right\| \\
& +\left(\alpha_{n, 2} \lambda+\alpha_{n, 2} \alpha_{n, 3} \lambda^{2}+\alpha_{n, 2} \alpha_{n, 3} \alpha_{n, 4} \lambda^{3}+\cdots+\prod_{i=2}^{p-1} \alpha_{n, i} \lambda^{p-2}\right) \Gamma \\
& +\left(\beta_{n, 2}+\alpha_{n, 2} \beta_{n, 3} \lambda+\alpha_{n, 2} \alpha_{n, 3} \beta_{n, 4} \lambda^{2}+\cdots+\prod_{i=2}^{p-1} \alpha_{n, i} \beta_{n, p} \lambda^{p-2}\right) \Gamma
\end{aligned}
$$


Thus, applying (6.4) and (6.13), one has

$$
\begin{aligned}
& \left\|z_{n+1}-z\right\| \leq\left(1-\alpha_{n, 1}-\beta_{n, 1}+\alpha_{n, 1}\left(1-\alpha_{n, 2}-\beta_{n, 2}\right) \lambda+\alpha_{n, 1} \alpha_{n, 2}\left(1-\alpha_{n, 3}-\beta_{n, 3}\right) \lambda^{2}\right. \\
& \left.+\cdots+\prod_{i=1}^{p-1} \alpha_{n, i}\left(1-\alpha_{n, p}-\beta_{n, p}\right) \lambda^{p-1}+\prod_{i=1}^{p} \alpha_{n, i} \lambda^{p-1} L \vartheta\right)\left\|z_{n}-z\right\| \\
& +\alpha_{n, 1}\left\|e_{n, 1}^{\prime}\right\|+\alpha_{n, 1} \alpha_{n, 2} \lambda\left\|e_{n, 2}^{\prime}\right\|+\alpha_{n, 1} \alpha_{n, 2} \alpha_{n, 3} \lambda^{2}\left\|e_{n, 3}^{\prime}\right\|+\cdots+\prod_{i=1}^{p} \alpha_{n, i} \lambda^{p-1}\left\|e_{n, p}^{\prime}\right\| \\
& +\prod_{i=1}^{p} \alpha_{n, i} \lambda^{p-1} L \vartheta b_{n}+\left\|e_{n, 1}^{\prime \prime}\right\|+\alpha_{n, 1} \lambda\left\|e^{\prime \prime}{ }_{n, 2}\right\|+\alpha_{n, 1} \alpha_{n, 2} \lambda^{2}\left\|e^{\prime \prime}{ }_{n, 3}\right\|+\cdots+\prod_{i=1}^{p-1} \alpha_{n, i} \lambda^{p-1}\left\|e^{\prime \prime}{ }_{n, p}\right\| \\
& +\left\|r_{n, 1}\right\|+\alpha_{n, 1} \lambda|| r_{n, 2}\left\|+\alpha_{n, 1} \alpha_{n, 2} \lambda^{2}\right\| r_{n, 3}\left\|+\cdots+\prod_{i=1}^{p-1} \alpha_{n, i} \lambda^{p-1}\right\| r_{n, p} \| \\
& +\left(\alpha_{n, 1} \lambda+\alpha_{n, 1} \alpha_{n, 2} \lambda^{2}+\alpha_{n, 1} \alpha_{n, 2} \alpha_{n, 3} \lambda^{3}+\cdots+\prod_{i=1}^{p-1} \alpha_{n, i} \lambda^{p-1}\right) \Gamma \\
& +\left(\beta_{n, 1}+\alpha_{n, 1} \beta_{n, 2} \lambda+\alpha_{n, 1} \alpha_{n, 2} \beta_{n, 3} \lambda^{2}+\cdots+\prod_{i=1}^{p-1} \alpha_{n, i} \beta_{n, p} \lambda^{p-1}\right) \Gamma \\
& \leq\left(1-(1-L \vartheta) \prod_{i=1}^{p} \alpha_{n, i} \lambda^{p-1}\right)\left\|z_{n}-z\right\|+\sum_{i=1}^{p} \prod_{j=1}^{i} \alpha_{n, j} \lambda^{i-1}\left\|e_{n, i}^{\prime}\right\| \\
& +\prod_{i=1}^{p} \alpha_{n, i} \lambda^{p-1} L \vartheta b_{n}+\sum_{i=2}^{p} \prod_{j=1}^{i-1} \alpha_{n, j} \lambda^{i-1}\left\|e^{\prime \prime}{ }_{n, i}\right\|+\sum_{i=2}^{p} \prod_{j=1}^{i-1} \alpha_{n, j} \lambda^{i-1}\left\|r_{n, i}\right\| \\
& +\left\|e_{n, 1}^{\prime \prime}\right\|+\left\|r_{n, 1}\right\|+\left(\sum_{i=2}^{p} \prod_{j=1}^{i-1} \alpha_{n, j} \lambda^{i-1}+\sum_{i=2}^{p} \prod_{j=1}^{i-1} \alpha_{n, j} \beta_{n, i} \lambda^{i-1}+\beta_{n, 1}\right) \Gamma \\
& \leq\left(1-(1-L \vartheta) \prod_{i=1}^{p} \alpha_{n, i} \lambda^{p-1}\right)\left\|z_{n}-z\right\|+(1-L \vartheta) \prod_{i=1}^{p} \alpha_{n, i} \lambda^{p-1} \frac{\frac{\sum_{i=1}^{p} \prod_{j=1}^{i} \alpha_{n, j} \lambda^{i-1}\left\|e_{n, i}^{\prime}\right\|}{\alpha \lambda^{\prime-1}}+L \vartheta b_{n}}{1-L \vartheta} \\
& +\sum_{i=2}^{p} \prod_{j=1}^{i-1} \alpha_{n, j} \lambda^{i-1}\left\|e_{n, i}^{\prime \prime}\right\|+\sum_{i=2}^{p} \prod_{j=1}^{i-1} \alpha_{n, j} \lambda^{i-1}\left\|r_{n, i}\right\|+\left\|e_{n, 1}^{\prime \prime}\right\|+\left\|r_{n, 1}\right\| \\
& +\left(\sum_{i=2}^{p} \prod_{j=1}^{i-1} \alpha_{n, j} \lambda^{i-1}+\sum_{i=2}^{p} \prod_{j=1}^{i-1} \alpha_{n, j} \beta_{n, i} \lambda^{i-1}+\beta_{n, 1}\right) \Gamma \text {. }
\end{aligned}
$$

If $L \geq 1$, then $L \gamma<1$, where $\gamma$ is the same as in (3.17), implies that

$$
\sqrt{1-2 \tau+\iota^{2}}+\frac{r L}{r-r^{\prime}} \sqrt{\varpi^{2}-2 \rho \kappa+\rho^{2} \sigma^{2}}<1 \text {, }
$$

whence deduce that $L \vartheta<1$. For the case that $L<1$, it is plain that $L \vartheta<1$. In view of (4.3), we note that all the conditions of Lemma 4.7 hold and so, (6.14) and Lemma 4.7 guarantee that the sequence $\left\{z_{n}\right\}_{n=0}^{\infty}$ generated by Algorithm 6.1 converges strongly to the solution $z \in \mathcal{H}$ of the problem (5.1) and there is nothing to prove. This completes the proof.

As in the proof of Theorem 6.8, we can prove the convergence of iterative sequences generated by Algorithms 6.2-6.5 and we omit their proofs.

Theorem 6.9. Suppose that T, $f, g, \rho$ and $S$ are the same as in Theorem 6.8 and let all the conditions of Theorem 6.8 hold. If there exists a constant $\alpha>0$ such that $\prod_{i=1}^{p} \alpha_{n, i}>\alpha$ for each $n \geq 0$, then the iterative sequence $\left\{z_{n}\right\}_{n=0}^{\infty}$ generated by Algorithm 6.2 converges strongly to the only element of $\operatorname{EGNWHE}\left(K_{r}, T, f, g\right)$.

Theorem 6.10. Let T, $f, \rho$ and $S$ be the same as in Theorem 4.9 and let all the conditions of Theorem 4.9 hold. If there exists a constant $\alpha>0$ such that $\prod_{i=1}^{p} \alpha_{n, i}>\alpha$ for each $n \geq 0$, then the iterative sequence $\left\{z_{n}\right\}_{n=0}^{\infty}$ generated by Algorithm 6.3 converges strongly to the only element of $\operatorname{GNWHE}\left(K_{r}, T, f\right)$. 
Theorem 6.11. Let $T, g$ and $\rho$ be the same as in Theorem 3.5 and suppose that the conditions (a), (b) and (3.19) in Theorem 3.5 hold. Let $S: K_{r} \rightarrow K_{r}$ be a nearly uniformly L-Lipschitzian mapping with the sequence $\left\{b_{n}\right\}_{n=0}^{\infty}$ such that for any $u \in$ $\operatorname{GNRNVID}\left(K_{r}, T, g\right), g(u) \in \operatorname{Fix}(S)$. Further, let $L \tilde{\theta}<1$, where $\tilde{\theta}$ is the same as in Theorem 4.10. If there exists a constant $\alpha>0$ such that $\prod_{i=1}^{p} \alpha_{n, i}>\alpha$ for each $n \geq 0$, then the iterative sequence $\left\{z_{n}\right\}_{n=0}^{\infty}$ generated by Algorithm 6.4 converges strongly to the only element of $\operatorname{GNWHE}\left(K_{r}, T, g\right)$.

Theorem 6.12. Assume that T, $\rho$ and $S$ are the same as in Theorem 4.11 and let all the conditions of Theorem 4.11 hold. If there exists a constant $\alpha>0$ such that $\prod_{i=1}^{p} \alpha_{n, i}>\alpha$ for each $n \geq 0$, then the iterative sequence $\left\{z_{n}\right\}_{n=0}^{\infty}$ generated by Algorithm 6.5 converges strongly to the only element of $\operatorname{NWHE}\left(K_{r}, T\right)$.

\section{Some remarks}

In view of Definition 2.11, we note that the condition relaxed cocoercivity of the operator $T$ is weaker than the condition strongly monotonicity of $T$. In other words, the class of relaxed cocoercive operators is more general than the class of strongly monotone operators. In the present section, we shall show that unlike claims of Noor [38], Noor et al. [44], Qin and Noor [47], they studied the convergence analysis of the proposed iterative algorithms under the condition of strongly monotonicity, not the mild condition relaxed cocoercivity.

Noor [38] proposed the following three-step iterative algorithm and its special forms for finding a common element of two different sets of solutions of the fixed points of the nonexpansive mappings and the general variational inequalities (3.9) and studied convergence analysis of the suggested iterative algorithm under some conditions.

Algorithm 7.1. (Algorithm 2.1 [38]) For any $x_{0} \in \mathcal{H}$, compute the approximate solution $x_{n}$ by the iterative schemes

$$
\left\{\begin{array}{l}
z_{n}=\left(1-c_{n}\right) x_{n}+c_{n} S\left\{x_{n}-g\left(x_{n}\right)+P_{K}\left[g\left(x_{n}\right)-\rho T x_{n}\right]\right\}, \\
y_{n}=\left(1-b_{n}\right) x_{n}+b_{n} S\left\{z_{n}-g\left(z_{n}\right)+P_{K}\left[g\left(z_{n}\right)-\rho T z_{n}\right]\right\}, \\
x_{n+1}=\left(1-a_{n}\right) x_{n}+a_{n} S\left\{y_{n}-g\left(y_{n}\right)+P_{K}\left[g\left(y_{n}\right)-\rho T y_{n}\right]\right\},
\end{array}\right.
$$

where $a_{n}, b_{n}, c_{n} \in[0,1]$ for all $n \geq 0$ and $S$ is the nonexpansive operator.

Theorem 7.2. (Theorem 3.1 [38]) Let $K$ be a closed convex subset of a real Hilbert space $\mathcal{H}$. Let $T$ be a relaxed $(\gamma, r)$-cocoercive and $\mu$-Lipschitzian mapping of $K$ into $\mathcal{H}$. Let $g$ be a relaxed $\left(\gamma_{1}, r_{1}\right)$-cocoercive and $\mu_{1}$-Lipschitzian mapping of $K$ into $\mathcal{H}$ and $S$ be a nonexpansive mapping of $K$ into $K$ such that $F(S) \cap G \mathrm{GI}(K, T, g) \neq \varnothing$. Let $\left\{x_{n}\right\}$ be a sequence defined by Algorithm 7.1, for any initial point $x_{0} \in K$, with the following conditions:

$$
\left\|\rho-\frac{r-\gamma \mu^{2}}{\mu^{2}}\right\| \leq \frac{\sqrt{\left(r-\gamma \mu^{2}\right)^{2}-\mu^{2} k(2-k)}}{\mu^{2}}, \quad r>\gamma \mu^{2}+\mu \sqrt{k(2-k)}, \quad k<1,
$$

where

$$
k=2 \sqrt{1+2 \gamma_{1} \mu_{1}^{2}-2 r_{1}+\mu_{1}^{2}}
$$


$a_{n}, b_{n}, c_{n} \in[0,1]$ and $\sum_{n=0}^{\infty} a_{n}=\infty$, then $x_{n}$ obtained from Algorithm 7.1 converges strongly to $x^{*} \in F(S) \cap \mathrm{GVI}(K, T, g)$.

From $k=2 \sqrt{1+2 \gamma_{1} \mu_{1}^{2}-2 r_{1}+\mu_{1}^{2}}$, it follows that $2\left(r_{1}-\gamma_{1} \mu_{1}^{2}\right)<1+\mu_{1}^{2}$. Accordingly, the condition $2\left(r_{1}-\gamma_{1} \mu_{1}^{2}\right)<1+\mu_{1}^{2}$ should be added to the conditions of Theorem 7.2. On the other hand, the conditions $r>\gamma \mu^{2}+\mu \sqrt{k(2-k)}$ and $k<1$ imply that $r>\gamma \mu^{2}$. Since $T$ is $(\gamma, r)$-relaxed cocoercive and $\mu$-Lipschitz continuous, the condition $r>\gamma \mu^{2}$ guarantees that the operator $T$ is $\left(r-\gamma \mu^{2}\right)$-strongly monotone. Therefore, one can rewrite Theorem 7.2 as follows.

Theorem 7.3. Let $K$ be a closed convex subset of a real Hilbert space $\mathcal{H}$ and let $T$ be a $\xi$-strongly monotone and $\mu$-Lipschitzian mapping of $K$ into $\mathcal{H}$. Let $g$ be a $\xi_{1}$-strongly monotone and $\mu_{1}$-Lipschitzian mapping of $K$ into $\mathcal{H}$ and $S$ be a nonexpansive mapping of $K$ into $K$ such that $F(S) \cap \operatorname{GVI}(K, T, g) \neq \varnothing$. If the constant $\rho$ satisfies the following condition:

$$
\left\{\begin{array}{l}
\left|\rho-\frac{\xi^{2}}{\mu^{2}}\right| \leq \frac{\sqrt{\xi^{2}-\mu^{2} k(2-k)}}{\mu^{2}} \\
\xi>\mu \sqrt{k(2-k)} \\
k=2 \sqrt{1-2 \xi_{1}^{2}+\mu_{1}^{2}}<1 \\
2 \xi_{1}^{2}<1+\mu_{1}^{2}
\end{array}\right.
$$

and the sequence $\left\{a_{n}\right\}$ satisfies $k=2 \sqrt{1+2 \gamma_{1} \mu_{1}^{2}-2 r_{1}+\mu_{1}^{2}}$, then the iterative sequence $\left\{x_{n}\right\}$ generated by Algorithm 7.1 converges strongly to the only element of $F(S)$ $\cap \operatorname{GVI}(K, T, g)$.

Remark 7.4. Theorem 3.2 in [38] is stated with the condition relaxed cocoercivity of the operators $T$ and $g$. Similarly, by using the conditions of Theorem 3.2 [38], we note that the operators $T$ and $g$ are in fact strongly monotone. Hence, Theorem 3.2 [38] is proved with the condition strongly monotonicity of the operators $T$ and $g$ instead of the mild condition relaxed cocoercivity.

Noor et al. [44] presented the following iterative scheme and its special forms for finding the common element of the solution sets of the general variational inequalities (3.9) and the nonexpansive mappings.

$$
\begin{array}{rllll}
\text { Algorithm 7.5. } & \text { (Algorithm } 3.1 \quad[44]) & \text { For } & \text { g given } \\
T^{-1} P_{K_{r}} z+\rho^{-1} Q_{K_{r}} z=0 & \Leftrightarrow \rho T g^{-1} P_{K_{r}} z+Q_{K_{r}} z=0 \\
& \Leftrightarrow \rho T g^{-1} P_{K_{r}} z+z-f g^{-1} P_{K_{r}} z=0 \\
& \Leftrightarrow z=f g^{-1} P_{K_{r}} z-\rho T g^{-1} P_{K_{r}} z \\
& \Leftrightarrow z=f(u)-\rho T(u) .
\end{array}
$$

$[0,1]$, compute the approximate solution $z_{n+1}$ by the iterative schemes

$$
\left\{\begin{array}{l}
g\left(u_{n}\right)=S P_{K} z_{n \prime} \\
z_{n+1}=\left(1-a_{n}\right) z_{n}+a_{n}\left\{g\left(u_{n}\right)-\rho T u_{n}\right\},
\end{array}\right.
$$

where $S$ is a non-expansive operator.

They also studied convergence analysis of the suggested iterative algorithm under some conditions as follows:

Theorem 7.6. (Theorem 3.1 [44]) Let $K$ be a closed convex subset of a real Hilbert space $\mathcal{H}$. Let $T$ be a relaxed $(\gamma, r)$-cocoercive and $\mu$-Lipschitzian mapping. Let $g$ be a 
relaxed $\left(\gamma_{1}, r_{1}\right)$-cocoercive and $\mu_{1}$-Lipschitzian mapping of $K$ into $\mathcal{H}$ and $S$ be a nonexpansive mapping of $\mathcal{H}$ into $\mathcal{H}$ such that $F(S) \cap \operatorname{GWHE}(\mathcal{H}, T, g, S) \neq \emptyset$. Let $\left\{z_{n}\right\}$ be a sequence defined by Algorithm 7.5, for any initial point $z_{0} \in K$, with the following conditions:

$$
\begin{gathered}
\left\|\rho-\frac{r-\gamma \mu^{2}}{\mu^{2}}\right\| \leq \frac{\sqrt{\left(r-\gamma \mu^{2}\right)^{2}-\mu^{2} k(2-k)}}{\mu^{2}}, \\
r>\gamma \mu^{2}+\mu \sqrt{k(2-k)}, \quad k<1,
\end{gathered}
$$

where

$$
k=2 \sqrt{1+2 \gamma_{1} \mu_{1}^{2}-2 r_{1}+\mu_{1}^{2}}
$$

$a_{n} \in[0,1]$ and $k=2 \sqrt{1+2 \gamma_{1} \mu_{1}^{2}-2 r_{1}+\mu_{1}^{2}}$, then $z_{n}$ obtained from Algorithm 7.5 converges strongly to $z^{*} \in F(S) \cap \operatorname{GWHE}(\mathcal{H}, T, g, S)$.

As in Theorem 7.2, since $k=2 \sqrt{1+2 \gamma_{1} \mu_{1}^{2}-2 r_{1}+\mu_{1}^{2}}$, it follows that $2\left(r_{1}-\gamma_{1} \mu_{1}^{2}\right)<1+\mu_{1}^{2}$. Hence the condition $2\left(r_{1}-\gamma_{1} \mu_{1}^{2}\right)<1+\mu_{1}^{2}$ should be added to Theorem 7.6. Moreover, by using the conditions $r>\gamma \mu^{2}+\mu \sqrt{k(2-k)}$ and $k<1$, $(\gamma, r)$-relaxed cocoercivity and $\mu$-Lipschitz continuity of the operator $T$ and $\left(\gamma_{1}, r_{1}\right)$ relaxed cocoercivity and $\mu_{1}$-Lipschitz continuity of the operator $g$, we note that the operators $T$ and $g$ are $\left(r-\gamma \mu^{2}\right)$-strongly monotone and $\left(r_{1}-\gamma_{1} \mu_{1}^{2}\right)$-strongly monotone, respectively. Therefore, Theorem 7.6 collapses to the following theorem.

Theorem 7.7. Let $K$ be a closed convex subset of a real Hilbert space $\mathcal{H}$ and let $T: \mathcal{H} \rightarrow \mathcal{H}$ be a $\xi$-strongly monotone and $\mu$-Lipschitzian mapping. Let $g: \mathcal{H} \rightarrow \mathcal{H}$ be a $\xi_{1}$-strongly monotone and $\mu_{1}$-Lipschitzian mapping and $S: \mathcal{H} \rightarrow \mathcal{H}$ be a nonexpansive mapping such that $F(S) \cap \operatorname{GVI}(K, T, g) \neq \varnothing$. If the constant $\rho$ satisfies the following condition:

$$
\left\{\begin{array}{l}
\left|\rho-\frac{\xi^{2}}{\mu^{2}}\right| \leq \frac{\sqrt{\xi^{2}-\mu^{2} k(2-k)}}{\mu^{2}} \\
\xi>\mu \sqrt{k(2-k)} \\
k=2 \sqrt{1-2 \xi_{1}^{2}+\mu_{1}^{2}}<1 \\
2 \xi_{1}^{2}<1+\mu_{1}^{2}
\end{array}\right.
$$

and the sequence $\left\{a_{n}\right\}$ satisfies $\sum_{n=0}^{\infty} a_{n}=\infty$, then the iterative sequence $\left\{z_{n}\right\}$ generated by Algorithm 7.5 converges strongly to the only element of $F(S) \cap \operatorname{GWHE}(\mathcal{H}, T, g, S)$.

Therefore, Noor et al. [44] proved the strongly convergence of the iterative sequence $\left\{z_{n}\right\}$ generated by Algorithm 7.5, under the condition strongly monotonicity of the operators $T$ and $g$, not under the mild condition relaxed cocoercivity.

Qin and Noor [47] proposed the following iterative algorithm and its special forms for solving the general variational inequalities (3.9).

Algorithm 7.8. (Algorithm $2.1[47])$ For any $z_{0} \in K$, compute the sequence $\left\{z_{n}\right\}$ by the iterative processes 


$$
\left\{\begin{array}{l}
g\left(u_{n}\right)=S P_{K} z_{n}, \\
z_{n+1}=\left(1-\alpha_{n}\right) z_{n}+\alpha_{n}\left[g\left(u_{n}\right)-\rho A u_{n}\right], \quad \forall n \geq 0,
\end{array}\right.
$$

where $\left\{\alpha_{n}\right\}$ is a sequence in $[0,1]$ and $S$ is a nonexpansive mapping.

They studied convergence analysis of the suggested iterative algorithm under some conditions as follows.

Theorem 7.9. (Theorem 3.1 [47]) Let $K$ be a closed convex subset of a real Hilbert space $\mathcal{H}$. Let $g: \mathcal{H} \rightarrow \mathcal{H}$ be a relaxed $\left(u_{1}, v_{1}\right)$-cocoercive and $\mu_{1}$-Lipschitz continuous mapping, $A: \mathcal{H} \rightarrow \mathcal{H}$ be a relaxed $\left(u_{2}, v_{2}\right)$-cocoercive and $\mu_{2}$-Lipschitz continuous mapping and $S$ be a nonexpansive mapping from $K$ into itself such that $F(S) \neq \varnothing$. Let $\left\{z_{n}\right\},\left\{u_{n}\right\}$ and $\left\{g\left(u_{n}\right)\right\}$ be the sequences generated by Algorithm 7.8 and $\left\{\alpha_{n}\right\}$ be a sequence in $[0,1]$. Assume that the following conditions are satisfied:

(C1) $2 \theta_{1}+\theta_{2}<1$, where $\theta_{1}=\sqrt{1+\mu_{1}^{2}-2 v_{1}+2 u_{1} \mu_{1}^{2}}$ and $\theta_{2}=\sqrt{1+\rho^{2} \mu_{2}^{2}-2 \rho v_{2}+2 \rho u_{2} \mu_{2}^{2}} ;$

(C2) $\sum_{n=0}^{\infty} \alpha_{n}=\infty$.

Then the sequences $\left\{z_{n}\right\},\left\{u_{n}\right\}$ and $\left\{g\left(u_{n}\right)\right\}$ converge strongly to $z^{*} \in \operatorname{WHE}(\mathcal{H}, A, S), u^{*}$ $\in \mathrm{VI}(K, A)$ and $g\left(u^{*}\right) \in F(S)$, respectively.

From the condition (C1), it follows that $2\left(v_{1}-u_{1} \mu_{1}^{2}\right)<1+\mu_{1}^{2}$ and $2 \rho\left(v_{2}-u_{2} \mu_{2}^{2}\right)<1+\mu_{2}^{2}$. Therefore, these conditions should be added to Theorem 7.9. On the other hand, the condition (C1) implies that $v_{i}>u_{i} \mu_{i}^{2}$, for $i=1,2$. Because $g$ is $\left(u_{1}, v_{1}\right)$-relaxed cocoercive and $\mu_{1}$-Lipschitz continuous, the condition $v_{1}>u_{1} \mu_{1}^{2}$ guarantees $\left(v_{1}-u_{1} \mu_{1}^{2}\right)$-strongly monotonicity of the operator $g$. Similarly, from $\left(u_{2}, v_{2}\right)$ relaxed cocoercivity and $\mu_{2}$-Lipschitz continuity of the operator $A$ and the condition $v_{2}>u_{2} \mu_{2}^{2}$, it follows that the operator $A$ is $\left(v_{2}-u_{2} \mu_{2}^{2}\right)$-strongly monotone. Hence Theorem 7.9 reduces to the following theorem:

Theorem 7.10. Let $K$ be a closed convex subset of a real Hilbert space $\mathcal{H}$ and let $g: \mathcal{H} \rightarrow \mathcal{H}$ be a $\xi_{1}$-strongly monotone and $\mu_{1}$-Lipschitz continuous mapping, $A: \mathcal{H} \rightarrow \mathcal{H}$ be $\xi_{2}$-strongly monotone and $\mu_{2}$-Lipschitz continuous mapping and let $S$ be a nonexpansive mapping from $K$ into itself such that $F(S) \neq \varnothing$. Let $\left\{z_{n}\right\},\left\{u_{n}\right\}$ and $\{g$ $\left.\left(u_{n}\right)\right\}$ be sequences generated by Algorithm 7.8. If the following conditions hold:

(C1) $2 \theta_{1}+\theta_{2}<1$, where $\theta_{1}=\sqrt{1+\mu_{1}^{2}-2 \xi_{1}}$ and $\theta_{2}=\sqrt{1+\rho^{2} \mu_{2}^{2}-2 \rho \xi_{2}}$;

(C2) $2 \xi_{1}<1+\mu_{1}^{2}, 2 \rho \xi_{2}<1+\rho \mu_{1}^{2}$ and $\sum_{n=0}^{\infty} \alpha_{n}=\infty$,

then the iterative sequences $\left\{z_{n}\right\},\left\{u_{n}\right\}$ and $\left\{g\left(u_{n}\right)\right\}$ generated by Algorithm 7.5 converge strongly to $z^{*} \in \operatorname{WHE}(\mathcal{H}, A, S), u^{*} \in \operatorname{VI}(K, A)$ and $g\left(u^{*}\right) \in F(S)$, respectively.

Remark 7.11. (1) Qin and Noor in Remark 3.2 [47] claimed that Theorem 7.9 is obtained under the mild condition relaxed cocoercivity of the operators $g$ and $A$. But, in view of the above facts, their results are obtained under the condition strongly 
monotonicity of the operators $g$ and $A$ not under the mild condition relaxed cocoercivity.

(2) The operators $A$ and $g$ in Corollaries 3.3 and 3.4 [47] are relaxed cocoercive. But we note that the conditions of the aforesaid corollaries guarantee that the operators $A$ and $g$ in these corollaries are in fact strongly monotone. Accordingly, Corollaries 3.3 and 3.4 in [47] are stated with the condition strongly monotonicity of the operators $A$ and $g$ instead of the mild condition relaxed cocoercivity.

Remark 7.12. In view of the above facts, we note that Theorems 4.8 and 4.10 generalize and improve Theorem 3.1 in [38]. Theorems 4.8, 4.10 and 4.11 generalize and improve Theorem 3.2 in [38]. Theorems 6.8-6.11 improve and generalize Theorem 3.2 in [42], Theorem 3.1 in [44] and [47] and Corollaries 3.3 and 3.4 in [47].

\section{Conclusion}

In this paper, we have introduced and considered some new classes of extended general nonlinear regularized nonconvex variational inequalities and the extended general nonconvex Wiener-Hopf equations involving three different nonlinear operators. By the projection operator technique, we have established the equivalence between the extended general nonlinear regularized nonconvex variational inequalities and the fixed point problems as well as the extended general nonconvex Wiener-Hopf equations. Then by this equivalent formulation, we have discussed the existence and uniqueness theorem for solution of the problem of extended general nonlinear regularized nonconvex variational inequalities. This equivalence and a nearly uniformly Lipschitzian mapping $S$ are used to suggest and analyze some new perturbed $p$-step projection iterative algorithms with mixed errors for finding an element of the set of the fixed points of the nearly uniformly Lipschitzian mapping $S$ which is unique solution of the problem of extended general nonlinear regularized nonconvex variational inequalities. We have presented some remarks about established statements by Noor [38], Noor et al. [44], Qin and Noor [47] and also have shown that their statements are special cases of our results. Several special cases are also discussed. It is expected that the results proved in this paper may simulate further research regarding the numerical methods and their applications in various fields of pure and applied sciences.

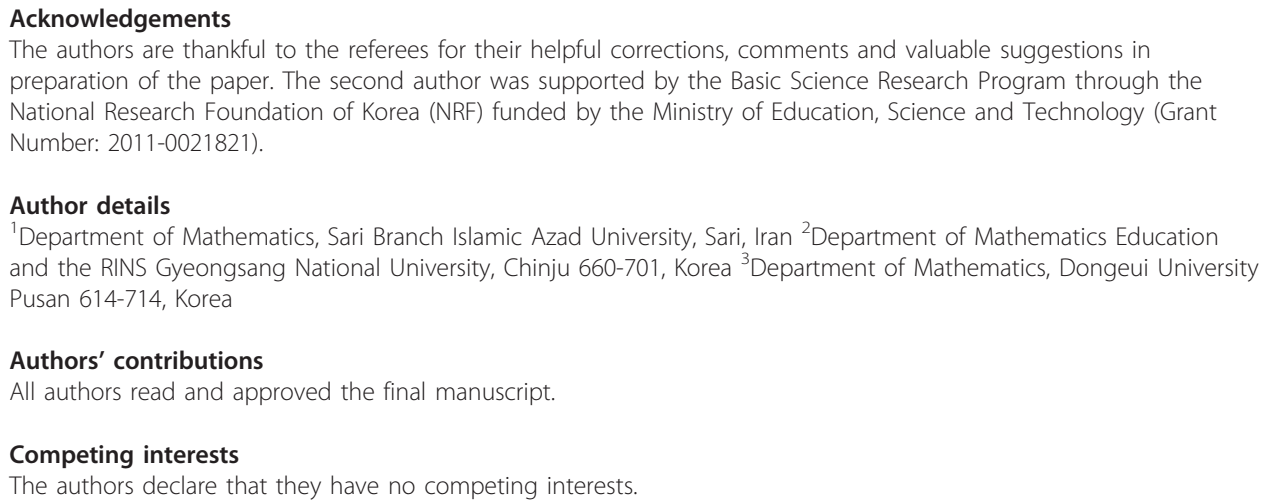




\section{References}

1. Stampacchia, G: Formes bilineaires coercitives sur les ensembles convexes. C R Acad Sci Paris 258, 4413-4416 (1964). 1, 3

2. Bensoussan, A, Lions, JL: Application des Inéquations variationelles en control et en Stochastiques. Dunod, Paris (1978). 1

3. Harker, PT, Pang, JS: Finite-dimensional variational inequality and nonlinear complementarity problems: a survey of theory, algorithm and applications. Math Program 48, 161-220 (1990). 1. doi:10.1007/BF01582255

4. Alimohammady, M, Balooee, J, Cho, YJ, Roohi, M: A new system of nonlinear fuzzy variational inclusions involving ( $A$, ๆ)-accretive mappings in uniformly smooth Banach spaces. J Inequal Appl 2009 (2009). Article ID 806727, 33 pages doi:10.1155/2010/806727

5. Alimohammady, M, Balooee, J, Cho, YJ, Roohi, M: Generalized nonlinear random equations with random fuzzy and relaxed cocoercive mappings in Banach spaces. Advan in Nonlinear Variat Inequal. 13, 37-58 (2010)

6. Alimohammady, M, Balooee, J, Cho, YJ, Roohi, M: Iterative algorithms for a new class of extended general nonconvex set-valued variational inequalities. Nonlinear Anal. 73, 3907-3923 (2010). doi:10.1016/j.na.2010.08.022

7. Alimohammady, M, Balooee, J, Cho, YJ, Roohi, M: New perturbed finite step iterative algorithms for a system of extended generalized nonlinear mixed quasi-variational inclusions. Comput Math Appl. 60, 2953-2970 (2010). doi:10.1016/j.camwa.2010.09.055

8. Agarwal, RP, Cho, YJ, Petrot, N: Systems of general nonlinear set-valued mixed variational inequalities problems in Hilbert spaces. Fixed Point Theory Appl 2011 (2011). 2011:31 doi:10.1186/1687-1812-2011-31

9. Cho, YJ, Lan, HY: A new class of generalized nonlinear multi-valued quasi-variational-like-inclusions with $\mathrm{H}$-monotone mappings. Math Inequal Appl. 10, 389-401 (2007)

10. Cho, YJ, Qin, $X$, Shang, MJ, Su, YF: Generalized nonlinear variational inclusions involving $(A, \eta)$-monotone mappings in Hilbert spaces. Fixed Point Theory and Appl 2007 (2007). Article ID 29653, 6 pages

11. Cho, YJ, Lan, HY: Generalized nonlinear random $(A, \eta)$-accretive equations with random relaxed cocoercive mappings in Banach spaces. Comput Math Appl. 55, 2173-2182 (2008). doi:10.1016/j.camwa.2007.09.002

12. Cho, YJ, Qin, X: Systems of generalized nonlinear variational inequalities and its projection methods. Nonlinear Anal. 69, 4443-4451 (2008). doi:10.1016/.na.2007.11.001

13. Cho, YJ, Qin, X: Generalized systems for relaxed cocoercive variational inequalities and projection methods in Hilbert spaces. Math Inequal Appl. 12, 365-375 (2009)

14. Cho, YJ, Petrot, N: Approximate solvability of a system of nonlinear relaxed cocoercive variational inequalities and Lipschitz continuous mappings in Hilbert spaces. Advan in Nonlinear Variat Inequal. 13, 91-101 (2010)

15. Cho, YJ, Argyros, IK, Petrot, N: Approximation methods for common solutions of generalized equilibrium, systems of nonlinear variational inequalities and fixed point problems. Comput Math Appl. 60, 2292-2301 (2010). doi:10.1016/j. camwa.2010.08.021

16. Lan, HY, Kang, Jl, Cho, YJ: Nonlinear ( $A, \eta)$-monotone operator inclusion systems involving non-monotone set-valued mappings. Taiwan J Math. 11, 683-701 (2007)

17. Qin, X, Kang, Jl, Cho, YJ: On quasi-variational inclusions and asymptotically pseudo-contractions. J Nonlinear Convex Anal. 11, 441-453 (2010)

18. Yao, Y, Cho, YJ, Liou, Y: Algorithms of common solutions for variational inclusions, mixed equilibrium problems and fixed point problems. Europ J Operat Res. 212, 242-250 (2011). doi:10.1016/j.jor.2011.01.042

19. Bnouhachem, A, Noor, MA: Numerical methods for general mixed variational inequalities. Appl Math Comput. 204, 27-36 (2008). doi:10.1016/j.amc.2008.05.134

20. Bounkhel, M: Existence results of nonconvex differential inclusions. Port Math (NS) 59(3):283-309 (2002). 2

21. Bounkhel, M: General existence results for second order nonconvex sweeping process with unbounded perturbations Port Math (NS) 60(3):269-304 (2003). 2

22. Bounkhel, M, Azzam, L: Existence results on the second order nonconvex sweeping processes with perturbations. Setvalued Anal 12(3):291-318 (2004). 2

23. Bounkhel, M, Tadji, L, Hamdi, A: Iterative schemes to solve nonconvex variational problems. J Inequal Pure Appl Math 4, $1-14$ (2003). 1

24. Bounkhel, M, Thibault, L: Further characterizations of regular sets in Hilbert spaces and their applications to nonconvex sweeping process. Preprint, Centro de Modelamiento Matematico (CMM). Universidad de Chile (2000). 2

25. Canino, A: On p-convex sets and geodesics. J Diff Equ 75, 118-157 (1988). 2. doi:10.1016/0022-0396(88)90132-5

26. Chang, SS, Cho, YJ, Zhou, H: Iterative Methods for Nonlinear Operator Equations in Banach Spaces. pp. xiv+459. Nova Science Publishers Inc., Huntington, NY (2002) ISBN: 1-59033-170-2 2.19

27. Clarke, FH: Optimization and Nonsmooth Analysis. Wiley, New York (1983). 2, 2.7

28. Clarke, FH, Ledyaev, YuS, Stern, RJ, Wolenski, PR: Nonsmooth Analysis and Control Theory. Springer, New York (1998). 1, $2,2,2,2.7$

29. Clarke, FH, Stern, RJ, Wolenski, PR: Proximal smoothness and the lower $C^{2}$ property. J Convex Anal 2(1/2):117-144 (1995). 1, 2, 2, 2.8, 2, 2.9, 2

30. Lions, PL, Mercier, B: Splitting algorithms for the sum of two nonlinear operators. SIAM J Numer Anal 16, 964-979 (1979). 5.1. doi:10.1137/0716071

31. Lions, JL, Stampacchia, G: Variational inequalities. Comm Pure Appl Math 20, 493-512 (1967). 1, 5.1. doi:10.1002/ cpa.3160200302

32. Liu, LS: Ishikawa and Mann iterative process with errors for nonlinear strongly accretive mappings in Banach spaces. J Math Anal Appl 194, 114-125 (1995). 4. doi:10.1006/jmaa.1995.1289

33. Noor, MA: Auxiliary principle technique for extended general variational inequalities. Banach J Math Anal 2, 33-39 (2008). 3

34. Noor, MA: Differentiable nonconvex functions and general variational inequalities. Appl Math Comput 199, 623-630 (2008). 3. doi:10.1016/j.amc.2007.10.023

35. Noor, MA: Iterative methods for general nonconvex variational inequalities. Albanian J Math 3(1):117-127 (2009). 5, 5.1

36. Noor, MA: Iterative schemes for nonconvex variational inequalites. J Optim Theory Appl 121, 385-395 (2004). 1

37. Noor, MA: General variational inequalities. Appl Math Lett 1(2):119-122 (1988). 3. doi:10.1016/0893-9659(88)90054-7 
38. Noor, MA: General variational inequalities and nonexpansive mappings. J Math Anal Appl 331, 810-822 (2007). 1, 4.6, 7, 7.1, 7.2, 7.4, 7.12, 8. doi:10.1016/j.jmaa.2006.09.039

39. Noor, MA: Sensitivity analysis of extended general variational inequalities. Appl Math E-Notes 9, 17-26 (2009). 3, 5, 5.1

40. Noor, MA: Some developments in general variational inequalities. Appl Math Comput 152, 199-277 (2004). 5. doi:10.1016/50096-3003(03)00558-7

41. Noor, MA: Variational Inequalities and Applications. Lecture Notes, Mathematics Department, COMSATS Institute of information Technology, Islamabad, Pakistan (2007-2009). 1

42. Noor, MA: Wiener-Hopf equations and variational inequalities. J Optim Theory Appl 79(1):197-206 (1993). 6.7, 7.12. doi:10.1007/BF00941894

43. Noor, MA, Huang, Z: Three-step iterative methods for nonexpansive mappings and variational inequalities. Appl Math Comput 187, 680-685 (2007). 1. doi:10.1016/j.amc.2006.08.088

44. Noor, MA, Zainab, S, Yaqoob, H: General Winer-Hope equations and nonexpansive mappings. J Math Inequal 2(2):215-227 (2008). 1, 4.6, 6.7, 7, 7, 7.5, 7.6, 7, 7.12, 8

45. Pang, LP, Shen, J, Song, HS: A modified predictor-corrector algorithm for solving nonconvex generalized variational inequalities. Comput Math Appl 54, 319-325 (2007). 1. doi:10.1016/j.camwa.2006.07.010

46. Poliquin, RA, Rockafellar, RT, Thibault, L: Local differentiability of distance functions. Trans Am Math Soc 352, 5231-5249 (2000). 1, 2, 2.7, 2, 2. doi:10.1090/50002-9947-00-02550-2

47. Qin, X, Noor, MA: General Wiener-Hopf equation technique for nonexpansive mappings and general variational inequalities in Hilbert spaces. Appl Math Comput 201, 716-722 (2008). 1, 6.7, 7, 7, 7.8, 7.9, 7.11, 7.12, 8. doi:10.1016/j. amc.2008.01.007

48. Robinson, SM: Normal maps induced by linear transformations. Math Oper Res 17, 691-714 (1992). 1. doi:10.1287/ moor.17.3.691

49. Robinson, SM: Sensitivity analysis of variational inequalities by normal-map techniques. In: Giannessi F, Maugeri A (eds.) Variational Inequalities and Network Equilibrium Problems. pp. 257-276. Plenum Press, New York (1995)

50. Shi, P: Equivalence of variational inequalities with Wiener-Hopf equations. Proc Am Math Soc 111, 339-346 (1991). 1, 5, 5.1. doi:10.1090/50002-9939-1991-1037224-3

51. Shi, P: An iterative method for obstacles problems via Green's functions. Nonlinear anal 15, 339-344 (1990). 1. doi:10.1016/0362-546X(90)90142-4

52. Sellami, H, Robinson, SM: Implementation of a continuation method for normal maps. Math Program. 76, 563-578 (1997)

53. Sahu, DR: Fixed points of demicontinuous nearly Lipschitzian mappings in Banach spaces. Comment Math Univ Carolin 46(4):653-666 (2005). 1, 2.12, 2.13

54. Goebel, K, Kirk, WA: A fixed point theorem for asymptotically nonexpansive mappings. Proc Am Math Soc 35, 171-174 (1972). 2.12. doi:10.1090/50002-9939-1972-0298500-3

55. Kirk, WA, Xu, HK: Asymptotic pointwise contractions. Nonlinear Anal 69, 4706-4712 (2008). 2.12. doi:10.1016/j. na.2007.11.023

doi:10.1186/1687-1812-2011-86

Cite this article as: Balooee et al:: The Wiener-Hopf Equation Technique for Solving General Nonlinear Regularized Nonconvex Variational Inequalities. Fixed Point Theory and Applications 2011 2011:86.

\section{Submit your manuscript to a SpringerOpen ${ }^{\odot}$ journal and benefit from:}

- Convenient online submission

- Rigorous peer review

- Immediate publication on acceptance

- Open access: articles freely available online

- High visibility within the field

- Retaining the copyright to your article

Submit your next manuscript at $\gg$ springeropen.com 